\title{
Gravitino dark matter and general neutralino NLSP
}

\author{
L. Covi, ${ }^{a}$ J. Hasenkamp, ${ }^{a, b}$ S. Pokorski ${ }^{c}$ and J. Roberts ${ }^{d}$ \\ ${ }^{a}$ Deutsches Elektronen-Synchrotron DESY, \\ Hamburg, Germany \\ ${ }^{b}$ Institute for Theoretical Physics, Hamburg University, \\ Hamburg, Germany \\ ${ }^{c}$ Institute of Theoretical Physics, Warsaw University, \\ Warsaw, Poland \\ ${ }^{d}$ Center for Cosmology and Particle Physics, New York University, \\ New York, NY 10003, U.S.A. \\ E-mail: Laura.Covi@desy.de, Jasper.Hasenkamp@desy.de, \\ Stefan.Pokorski@fuw.edu.pl, Jonathan.Roberts@nyu.edu
}

ABSTRACT: We study the scenario of gravitino DM with a general neutralino NLSP in a model independent way. We consider all neutralino decay channels and compare them with the most recent BBN constraints. We check how those bounds are relaxed for a Higgsino or a Wino NLSP in comparison to the Bino neutralino case and look for possible loopholes in the general MSSM parameter space. We determine constraints on the gravitino and neutralino NLSP mass and comment on the possibility of detecting these scenarios at colliders.

KEYwords: Cosmology of Theories beyond the SM, Supersymmetric Standard Model ARXiv EPRINT: 0908.3399 


\section{Contents}

1 Introduction 1

2 Neutralino mass matrix and SUSY breaking parameters 3

3 Neutralino NLSP decays 3

3.1 Primary Bino decay channels 4

3.2 Primary Wino decay channels 5

3.3 Primary Higgsino decay channels 5

$\begin{array}{ll}3.4 & \text { Gaugino hadronic branching ratios }\end{array}$

3.5 Higgsino hadronic branching ratios 9

4 Neutralino thermal abundance and BBN $\quad 11$

$\begin{array}{lll}4.1 & \text { Bino-Wino NLSP } & 12\end{array}$

$\begin{array}{llr}4.2 & \text { Bino-Higgsino NLSP } & 16\end{array}$

$\begin{array}{lll}4.3 & \text { Wino-Higgsino NLSP } & 18\end{array}$

$\begin{array}{llr}5 & \text { General neutralino NLSP } & 19\end{array}$

6 Conclusions $\quad 22$

\section{Introduction}

The gravitino was the first supersymmetric Dark Matter (DM) candidate [1] and is still one of the best motivated today. In some of the supersymmetry breaking schemes, such as those that rely on gauge [2] or even gaugino mediation [3], such a state is either automatically, or in the second case very naturally [4], the lightest supersymmetric particle. An equally important motivation is thermal leptogenesis: producing the lepton asymmetry without stretching parameters requires a relatively high reheating temperature around $10^{10} \mathrm{GeV}$ [5], which cannot be reconciled with an unstable gravitino unless it is very heavy or so light as to be irrelevant for the energy budget of the universe. In general the gravitino energy density from thermal scatterings depends on the supersymmetric parameters and the temperature as $[6]$

$$
\Omega_{3 / 2} h^{2} \simeq 0.3\left(\frac{100 \mathrm{GeV}}{m_{3 / 2}}\right)\left(\frac{m_{\tilde{g}}}{1 \mathrm{TeV}}\right)^{2}\left(\frac{T_{R}}{10^{10} \mathrm{GeV}}\right)
$$

This result arises from the fact that the gravitino interactions in the goldstino limit are inversely proportional to its mass and therefore larger gravitino masses correspond to more weakly interacting gravitinos and thus lower number densities. Therefore a heavy stable 
gravitino does allow for a large reheating temperature, but it also implies a long lifetime for the Next-to-Lightest-Supersymmetric-Particle (NLSP) if R-parity is conserved. This in turn risks a clash between NLSP decay and Big Bang Nucleosynthesis (BBN) [7]. In this work we will investigate which mass ranges are still consistent with a gravitino LSP as DM for a general neutralino NLSP.

Specific models like the CMSSM have been studied in [8] and the case of a general Bino NLSP has been considered with some approximations in $[9,10]$; in these papers it was found that it is very difficult to reconcile such scenarios with a gravitino mass above $1 \mathrm{GeV}$ or with the parametric equality $m_{3 / 2}=m_{0}$. We would like to see if this is true in the more general case. For a non-Bino neutralino we would naively expect the situation to improve due to the smaller number densities or due to the different branching ratios into hadrons.

The case of a Higgsino NLSP naturally has a larger annihilation cross-section, leading to smaller number densities. However in the CMSSM it arises only in the finely tuned focuspoint region, which corresponds to very large supersymmetric masses. If we relax some of the usual assumptions on universality, we can obtain a Higgsino NLSP at lower masses, in particular in more general models of gauge mediation of supersymmetry breaking [11]. A partially Higgsino NLSP can also be found in models with non-universal Higgs masses, such as in gaugino mediation [12].

A Wino (N)LSP has predominantly been studied in the context of anomaly mediation [13], where it is naturally one of the lightest particles. Unfortunately in those models the gravitino is always very heavy and cannot be the LSP. We will consider here the possibility of a Wino NLSP for the most general SUSY breaking parameters and in particular for low values of the gaugino mass parameter $M_{2}$ and non universal boundary conditions. A Wino NLSP can occur naturally in certain GUT models with F-terms which are nonsinglet under the GUT group [14]. Note that a Wino NLSP may be possible also in the case of $\mathrm{U}(1)^{\prime}$ mediated supersymmetry breaking [15].

The goal of this analysis is to close any gap in the study of the neutralino NLSP with gravitino DM scenario, either coming from simplifications in the computations of the NLSP number density or from approximations in its decay rates and hadronic branching ratio. For this purpose we compute and give the complete results for the two and three body neutralino decays.

This paper is organised as follows: after summarising the general neutralino properties in section 2, we will discuss its decays into gravitino and Standard Model particles in section 3, assuming it is the NLSP. We will discuss with particular care the hadronic channels since they tend to be more strongly constrained by Nucleosynthesis. We will then review the computation of the neutralino relic abundance in section 4 and then consider specifically the cases of Bino-Wino, Bino-Higgsino and Wino-Higgsino neutralino and compare them directly with the BBN constraints of reference [16]. Furthermore we will generalise our result for any neutralino mixing in section 5 and draw our conclusions concerning supersymmetry breaking parameters and specific models in section 6 . 


\section{Neutralino mass matrix and SUSY breaking parameters}

Supersymmetry and EW symmetry breaking produce a non-trivial mass matrix for the neutralino in the basis of the gaugino-Higgsino states, given in the Bino-Wino-Higgsino basis by [17]

$$
M=\left(\begin{array}{cccc}
M_{1} & 0 & -M_{Z} s_{W} c_{\beta} & M_{Z} s_{W} s_{\beta} \\
0 & M_{2} & M_{Z} c_{W} c_{\beta} & -M_{Z} c_{W} s_{\beta} \\
-M_{Z} s_{W} c_{\beta} & M_{Z} c_{W} c_{\beta} & 0 & \mu \\
M_{Z} s_{W} s_{\beta} & -M_{Z} c_{W} s_{\beta} & \mu & 0
\end{array}\right)
$$

where $M_{1}, M_{2}$ are the supersymmetric gaugino mass parameters for the $\mathrm{U}(1)$ and $\mathrm{SU}(2)$ sector respectively, $\mu$ is the supersymmetric Higgs mass parameter, $M_{Z}$ the mass of the $\mathrm{Z}$ gauge boson, $s / c_{W}=\sin / \cos \theta_{W}$ with $\theta_{W}$ being the Weinberg angle, while $s / c_{\beta}=$ $\sin / \cos \beta$ is determined by the ratio of the Higgs v.e.v.s as $\tan \beta=v_{u} / v_{d}$.

The mass eigenstates are mixed gaugino/Higgsino states and their mixing angles and masses are determined by the SUSY breaking parameters $M_{1}, M_{2}$ and $\tan \beta, \mu$. In the following we will consider these as free parameters. In general then the mass matrix above can be diagonalised by a unitary matrix $N_{i j}$ such that

$$
\chi_{i}^{0}=N_{i j} \Psi_{j}^{0}
$$

where $\Psi^{0}=\left(\tilde{B}, \tilde{W}^{0}, \tilde{H}_{d}^{0}, \tilde{H}_{u}^{0}\right)^{T}$. If we consider all the parameters in the mass matrix to be real, as we will do here, then the matrix $N$ can be chosen real and orthogonal. The particle we are interested in is the lightest neutralino $\chi_{1}^{0}$, which may naturally become the NLSP in many supersymmetry breaking scenarios with a gravitino LSP. As is clear from the mass matrix above, the values of the parameters $M_{1}, M_{2}, \mu$ (and more weakly $\tan \beta$ ) determine the composition of the lightest neutralino and therefore its decay channels and relic abundance. In the following we will take the neutralino mass parameters at the low scale as the inputs and not assume any special relation between them. In this way we will be able to explore the most general scenario with a neutralino NLSP. But first we discuss the decay channels for the neutralino gauge eigenstates.

\section{Neutralino NLSP decays}

In the case of conserved R-parity and gravitino LSP, the neutralino couples with gravitino and gauge boson or Higgs boson via the supergravity interactions [18]. In general for gravitino LSP the dominant couplings are those involving the Goldstino component of the gravitino and therefore they are enhanced by $m_{\tilde{\chi}} / m_{\frac{3}{2}}$. Therefore the neutralino lifetime always contains an overall factor $x_{\frac{3}{2}}^{2}=\left(m_{\frac{3}{2}} / m_{\tilde{\chi}}\right)^{2}$. In the following we will discuss first the decays for the neutralino pure states: Bino, Wino and Higgsino. 

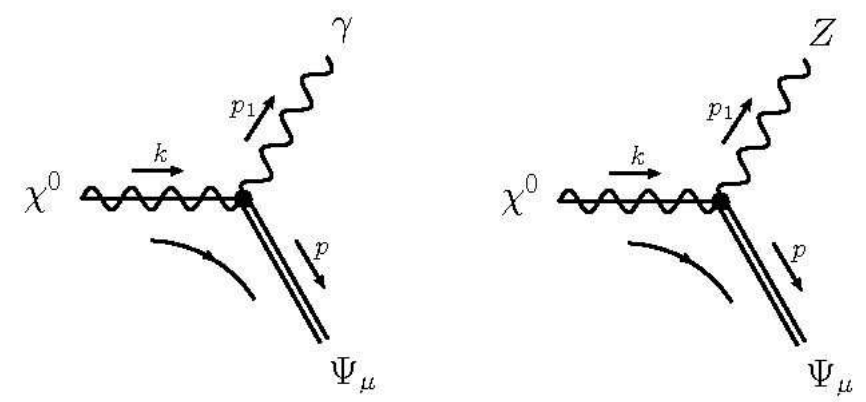

Figure 1. Feynman diagrams for the decay of a gaugino neutralino into neutral gauge bosons and gravitino.

\subsection{Primary Bino decay channels}

A Bino neutralino can decay into a photon and a gravitino via its photino component and the decay rate is given by

$$
\Gamma\left(\tilde{B} \rightarrow \psi_{3 / 2} \gamma\right)=\frac{\left|\cos \theta_{W}\right|^{2}}{48 \pi M_{P}^{2}} \frac{m_{\tilde{B}}^{3}}{x_{\frac{3}{2}}^{2}}\left(1-x_{\frac{3}{2}}^{2}\right)^{3}\left(1+3 x_{\frac{3}{2}}^{2}\right),
$$

where $\theta_{W}$ is the Weinberg angle, $m_{\tilde{B}}$ the Bino mass. For a negligible gravitino mass in the phase space factor, the decay of a pure Bino state is dominated by this channel and the lifetime can be approximated as:

$$
\Gamma^{-1}\left(\tilde{B} \rightarrow \psi_{3 / 2} \gamma\right)=7.7 \times 10^{4} \mathrm{~s}\left(\frac{m_{\tilde{B}}}{100 \mathrm{GeV}}\right)^{-5}\left(\frac{m_{\frac{3}{2}}}{1 \mathrm{GeV}}\right)^{2}
$$

If it is kinematically allowed, the Bino can also decay into a $\mathrm{Z}$ boson and a gravitino, the decay rate for this channel is given by

$$
\begin{aligned}
\Gamma\left(\tilde{B} \rightarrow \psi_{3 / 2} Z\right)= & \frac{\left|\sin \theta_{W}\right|^{2}}{48 \pi M_{P}^{2}} \frac{m_{\tilde{B}}^{3}}{x_{\frac{3}{2}}^{2}} \sqrt{1-2\left(x_{\frac{3}{2}}^{2}+x_{Z}^{2}\right)+\left(x_{Z}^{2}-x_{\frac{3}{2}}^{2}\right)^{2}} \\
& \times\left[\left(1-x_{\frac{3}{2}}^{2}\right)^{2}\left(1+3 x_{\frac{3}{2}}^{2}\right)-x_{Z}^{2}\left\{3+x_{\frac{3}{2}}^{3}\left(x_{\frac{3}{2}}-12\right)\right.\right. \\
& \left.\left.-x_{Z}^{2}\left(3-x_{\frac{3}{2}}^{2}-x_{Z}^{2}\right)\right\}\right]
\end{aligned}
$$

where here $x_{Z}=M_{Z} / m_{\tilde{B}}$.

So for large Bino masses the Bino lifetime is approximately given by

$$
\Gamma^{-1}\left(\tilde{B} \rightarrow \psi_{3 / 2} \gamma / Z\right)=57 \mathrm{~s}\left(\frac{m_{\tilde{B}}}{1 \mathrm{TeV}}\right)^{-5}\left(\frac{m_{\frac{3}{2}}}{10 \mathrm{GeV}}\right)^{2}
$$

so that it becomes shorter than $0.1 \mathrm{~s}$ for masses larger than $3.6 \mathrm{TeV} \times\left(\frac{m_{\frac{3}{2}}}{10 \mathrm{GeV}}\right)^{2 / 5}$. 


\subsection{Primary Wino decay channels}

A Wino neutralino can also decay into a photon and a gravitino via its photino component and the decay rate is given in this case by

$$
\Gamma\left(\tilde{W} \rightarrow \psi_{3 / 2} \gamma\right)=\frac{\left|\sin \theta_{W}\right|^{2}}{48 \pi M_{P}^{2}} \frac{m_{\tilde{W}}^{3}}{x_{\frac{3}{2}}^{2}}\left(1-x_{\frac{3}{2}}^{2}\right)^{3}\left(1+3 x_{\frac{3}{2}}^{2}\right)
$$

where $m_{\tilde{W}}$ is the Wino mass.

As with the Bino, the lifetime of a pure Wino state is dominated by this channel and for a negligible gravitino mass in the phase space factor it can be simplified to:

$$
\Gamma^{-1}\left(\tilde{W} \rightarrow \psi_{3 / 2} \gamma\right)=2.5 \times 10^{5} \mathrm{~s}\left(\frac{m_{\tilde{W}}}{100 \mathrm{GeV}}\right)^{-5}\left(\frac{m_{\frac{3}{2}}}{1 \mathrm{GeV}}\right)^{2}
$$

For a heavier Wino the channel into a $\mathrm{Z}$ boson and a gravitino opens up, with the decay rate

$$
\begin{aligned}
\Gamma\left(\tilde{W} \rightarrow \psi_{3 / 2} Z\right)= & \frac{\left|\cos \theta_{W}\right|^{2}}{48 \pi M_{P}^{2}} \frac{m_{\tilde{W}}^{3}}{x_{\frac{3}{2}}^{2}} \sqrt{1-2\left(x_{\frac{3}{2}}^{2}+x_{Z}^{2}\right)+\left(x_{Z}^{2}-x_{\frac{3}{2}}^{2}\right)^{2}} \\
& \times\left[\left(1-x_{\frac{3}{2}}^{2}\right)^{2}\left(1+3 x_{\frac{3}{2}}^{2}\right)-x_{Z}^{2}\left\{3+x_{\frac{3}{2}}^{3}\left(x_{\frac{3}{2}}-12\right)\right.\right. \\
& \left.\left.-x_{Z}^{2}\left(3-x_{\frac{3}{2}}^{2}-x_{Z}^{2}\right)\right\}\right],
\end{aligned}
$$

similar to the case of a Bino neutralino. We see clearly that the decay into $\mathrm{Z}$ is stronger for the Wino case than for the Bino and this immediately gives a stronger decay into hadrons. For a heavy Wino, the decay rate becomes equal to the Bino one, as given in eq. (3.4).

For large Wino masses the channel into a gravitino and a $\mathrm{W}$ pair opens up, via the Wino-gravitino-W-W 4-vertex, which arises from the non-abelian interaction, in addition to the corresponding three gauge bosons vertex and other diagrams with intermediate charginos and Higgs bosons. The main part of the contribution from the gauge sector, which appears at first sight to be enhanced by power of $m_{\tilde{W}}^{4} / M_{W}^{4}$ compared to the 2body decays, cancels out thanks to gauge invariance, as found also in [19]. We also found a cancellation of the sub-leading terms $m_{\tilde{W}}^{2} / M_{W}^{2}$, so this channel, suppressed by an additional $\alpha_{e m}$ and the 3 -body phase space always remains negligible.

\subsection{Primary Higgsino decay channels}

A pure Higgsino has direct decay channels into the scalar and pseudo-scalar Higgses and a gravitino, as well as into a $\mathrm{Z}$ boson and gravitino. In case the bosons are too heavy to be produced on-shell, the decay proceeds via off-shell Higgses mainly into heavy quarks, $b \bar{b}$, whilst the decay through an off-shell $\mathrm{Z}$ goes to both quarks and leptons. The decay rate 

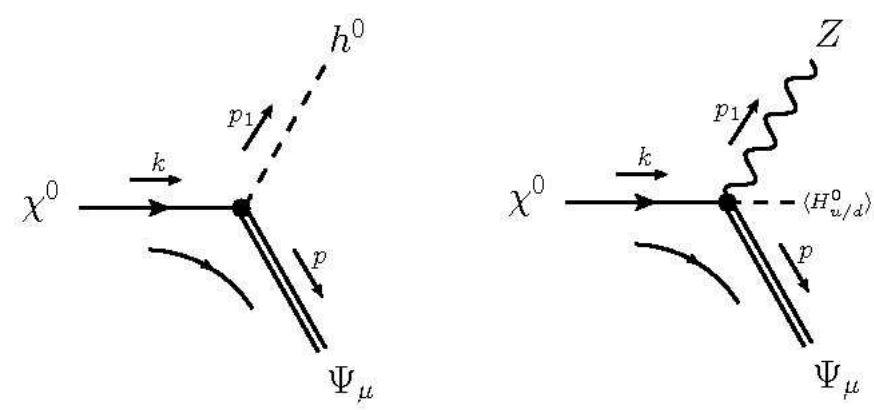

Figure 2. Feynman diagrams for the decay of a Higgsino neutralino into neutral Higgs or gauge bosons and gravitino.

into an on-shell $\mathrm{Z}$ boson is given by

$$
\begin{aligned}
\Gamma\left(\tilde{H} \rightarrow \psi_{3 / 2} Z\right)= & \frac{\left|-N_{13} \cos \beta+N_{14} \sin \beta\right|^{2}}{96 \pi M_{P}^{2}} \frac{m_{\tilde{H}}^{3}}{x_{\frac{3}{2}}^{2}} \sqrt{1-2\left(x_{\frac{3}{2}}^{2}+x_{Z}^{2}\right)+\left(x_{Z}^{2}-x_{\frac{3}{2}}^{2}\right)^{2}} \\
& \times\left[\left(1+x_{\frac{3}{2}}\right)^{2}\left(1-x_{\frac{3}{2}}\right)^{4}-x_{Z}^{2}\left\{\left(1-x_{\frac{3}{2}}\right)^{2}\left(3+2 x_{\frac{3}{2}}-9 x_{\frac{3}{2}}^{2}\right)\right.\right. \\
& \left.\left.-x_{Z}^{2}\left(3-2 x_{\frac{3}{2}}-9 x_{\frac{3}{2}}^{2}-x_{Z}^{2}\right)\right\}\right],
\end{aligned}
$$

where $N_{i j}$ is the neutralino mixing matrix and we consider a mixed Higgsino initial state, $m_{\tilde{H}}$ is the Higgsino mass and again $x_{a}=M_{a} / m_{\tilde{H}}$. The decay into light Higgs is instead

$$
\begin{aligned}
\Gamma\left(\tilde{H} \rightarrow \psi_{3 / 2} h\right)= & \frac{\left|-N_{13} \sin \alpha+N_{14} \cos \alpha\right|^{2}}{96 \pi M_{P}^{2}} \frac{m_{\tilde{H}}^{3}}{x_{\frac{3}{2}}^{2}} \sqrt{1-2\left(x_{\frac{3}{2}}^{2}+x_{h}^{2}\right)+\left(x_{h}^{2}-x_{\frac{3}{2}}^{2}\right)^{2}} \\
& \times\left[\left(1-x_{\frac{3}{2}}\right)^{2}\left(1+x_{\frac{3}{2}}\right)^{4}-x_{h}^{2}\left\{\left(1+x_{\frac{3}{2}}\right)^{2}\left(3-2 x_{\frac{3}{2}}+3 x_{\frac{3}{2}}^{2}\right)\right.\right. \\
& \left.\left.-x_{h}^{2}\left(3+2 x_{\frac{3}{2}}+3 x_{\frac{3}{2}}^{2}-x_{h}^{2}\right)\right\}\right],
\end{aligned}
$$

where $\alpha$ is the mixing angle between the two real parts of the Higgs bosons $H_{u}$ and $H_{d}$ into the $h$ and $\mathrm{H}$ mass eigenstates.

For large neutralino masses the decays into the heavy Higgs bosons open up. The heavy scalar Higgs channel is given by the above expression (3.9), but with the overall prefactor given by the orthogonal combination $\left|N_{13} \cos \alpha+N_{14} \sin \alpha\right|^{2}$ and $x_{h} \rightarrow x_{H}$, while the decay to the pseudo-scalar Higgs has the same form as the decay into the $\mathrm{Z}$ boson in eq. (3.8), but with the overall prefactor $\left|N_{13} \sin \beta+N_{14} \cos \beta\right|^{2}$ and $x_{Z} \rightarrow x_{A}$. We will not consider these decays further since they remain sub-dominant apart from the case when the neutralino composition is such to suppress the light Higgs channel.

In the decoupling limit $\sin \alpha=-\cos \beta$ and $\cos \alpha=\sin \beta$, and for $N_{13}=N_{14}$, the decay time of a Higgsino neutralino is given by

$$
\Gamma^{-1}\left(\tilde{H} \rightarrow \psi_{3 / 2} h / Z\right)=114 \mathrm{~s}\left(\frac{m_{\tilde{H}}}{1 \mathrm{TeV}}\right)^{-5}\left(\frac{m_{\frac{3}{2}}}{10 \mathrm{GeV}}\right)^{2}
$$


so that it becomes shorter than $0.1 \mathrm{~s}$ for masses larger than $4.1 \mathrm{TeV} \times\left(\frac{m_{\frac{3}{2}}}{10 \mathrm{GeV}}\right)^{2 / 5}$.

Note that for large Higgsino masses a supergravity 4-vertex may become important. In this case it is the Higgsino-gravitino- $h$-Z 4 -vertex, which arises from the Higgs scalar derivative coupling, and allows the direct decay into a gravitino, light Higgs and a Z. An analogous final state is also obtained by Higgs radiation from an intermediate Z, Z radiation from a pseudo-scalar Higgs or via neutralino intermediate states. In this case again an important cancellation of terms of order $m_{\tilde{H}}^{2} / M_{Z}^{2}$ takes place between the different diagrams thanks to gauge invariance and so this channel is always sub-dominant.

\subsection{Gaugino hadronic branching ratios}

The gaugino decay into hadrons proceeds via an intermediate off-shell photon, via an off/on-shell Z-boson and also via intermediate off-shell squarks. The decay via intermediate photon is particularly simple in the limit of near massless quarks. For the up quark at leading order in $x_{\frac{3}{2}}$ and $m_{u}$ this is given by:

$$
\Gamma\left(\tilde{\chi}_{G} \rightarrow \psi_{3 / 2} \gamma^{*} \rightarrow \psi_{3 / 2} u \bar{u}\right)=\frac{\left|N_{11} \cos \theta_{W}+N_{12} \sin \theta_{W}\right|^{2}}{27(2 \pi)^{3} M_{P}^{2}} \frac{m_{\tilde{\chi}_{G}}^{3}}{x_{\frac{3}{2}}^{2}} \log \left(\frac{m_{\tilde{\chi}_{G}}}{2 m_{u}}\right) .
$$

Here we see that the contribution is actually enhanced by a logarithmic IR divergence. Therefore the branching ratio into quarks never quite vanishes, always remaining greater than 0.03. This same logarithmic divergence ensures that the light quarks dominate the hadronic channels for low gaugino masses. Note that for late times in Nucleosynthesis, above $100 \mathrm{~s}$ or so, it is assumed that only quarks produced with energy above $2 \mathrm{GeV}$ are able to hadronise into nucleons and affect the BBN predictions as hadrons, while at lower energies they can only end up in light mesons that decay before interacting and produce mostly leptons or photons [20,21]. Therefore in figure 3 we also give the result with a $2 \mathrm{GeV}$ IR cut-off. We see that the introduction of such a scale only modifies the hadronic branching ratio at low neutralino masses and reduces it maximally by a factor three compared to the one computed with the physical quark masses. In general we find a hadronic branching ratio always much larger than $10^{-3}$, a value which is often taken as the reference minimal value for a Bino neutralino. To be conservative we will use the result obtained with the physical quark masses in our exclusion plots. Note that in the region of the parameter space in which the NLSP has a short lifetime, the effect of the interconversion of protons to neutrons from light mesons can be important [20]. For the case of an intermediate $\mathrm{Z}$ boson the IR divergence is not present and the contribution becomes largest for an on-shell $\mathrm{Z}$ with the branching ratio being at most equal to the decay into a $\mathrm{Z}$ boson weighted by the respective hadronic width as discussed in [9].

The channel with intermediate squarks has been neglected so far. It is clearly negligible for heavy squarks. We have implemented the full calculation of this contribution for varying squark masses. We find that in the limit of large universal squark masses this contribution reduces to

$$
\Gamma\left(\tilde{\chi}_{G} \rightarrow \psi_{3 / 2} \tilde{q} \rightarrow \psi_{3 / 2} q \bar{q}\right) \rightarrow \frac{\left|N_{11} \cos \theta_{W}+N_{12} \sin \theta_{W}\right|^{2}}{27(2 \pi)^{3} M_{P}^{2}} \frac{m_{\tilde{\chi}_{G}}^{7}}{m_{\tilde{q}}^{4} x_{\frac{3}{2}}^{2}},
$$




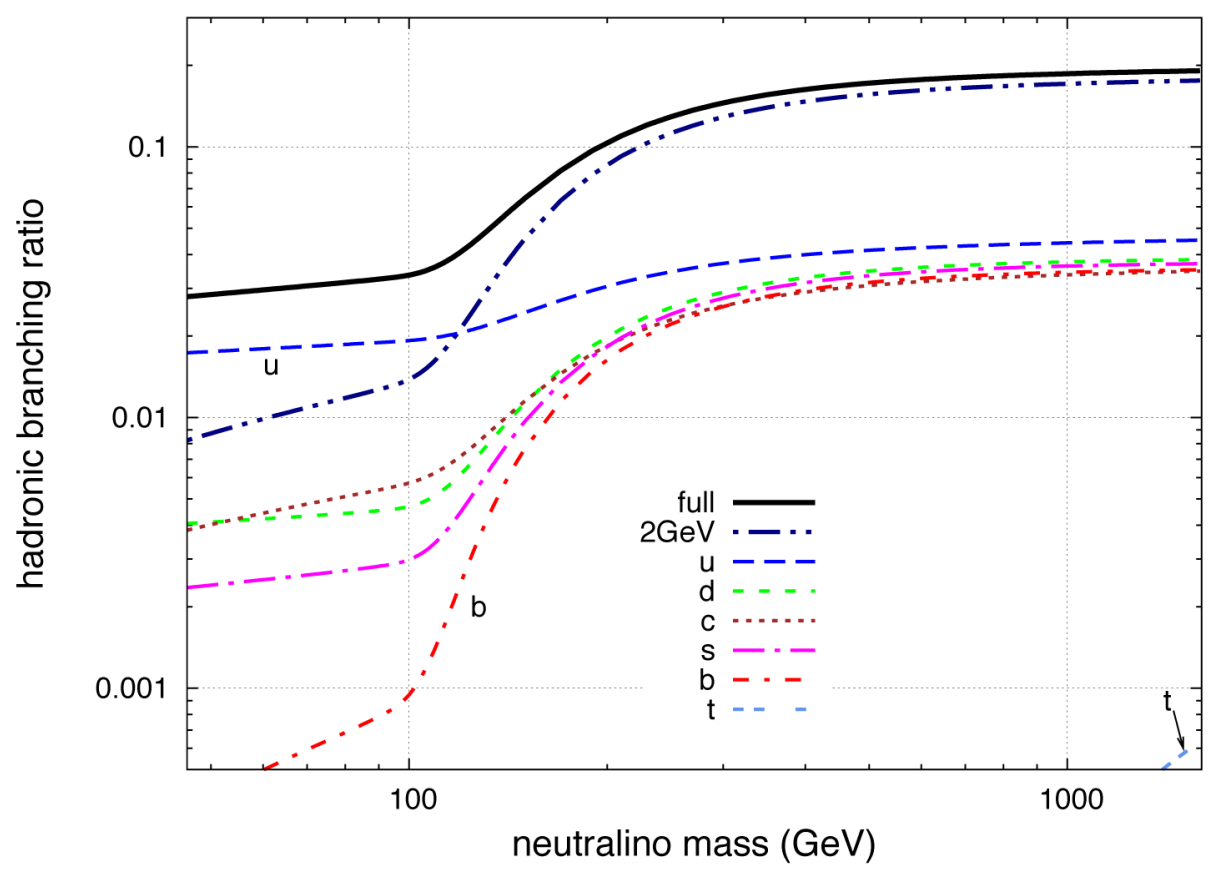

Figure 3. Branching ratios into a quark pair and gravitino for a Bino neutralino and negligible gravitino mass in the phase space factor. Below the $\mathrm{Z}$ threshold the decay is dominated by the off-shell photon and there the light quark channels are preferred due to the IR logarithmic enhancement. Above the $\mathrm{Z}$ threshold, the $\mathrm{Z}$ channel starts to become important and all light quarks are produced democratically. The top quark production is always negligible due to the strong phase space suppression, even for large neutralino masses.

and it is suppressed by at least a factor $\left(m_{\tilde{\chi}_{G}} / m_{\tilde{q}}\right)^{4}$ compared to the other channels. Surprisingly also for nearly degenerate masses between the squarks and the neutralino, the contribution of the squarks of the third generation amounts only to at most $1 \%$ of the hadronic branching ratio, due to the much stronger off-shell photon channel in the light quarks [18]. A larger relative correction of about $7 \%$ arises if the degenerate scalar is the superpartner of the up quark and the neutralino mass is below the $\mathrm{Z}$ threshold, but in that case the hadronic branching ratio is small, so the increase is anyway negligible. Moreover the mass degeneracy has to be pretty extreme, about $1 \%$, and then also coannihilation effects between the neutralino and the squarks become important. We therefore neglect these terms in the parameter scans of section 4.

We show the result of the full calculation of the branching ratio to the different quark final states for a pure Bino in figure 3. We see that, as expected, the light quarks dominate for small neutralino masses and that the top quark contribution is always negligible. Note that the hadronic branching ratio is practically independent of the gravitino mass since there is an overall factor of $x_{\frac{3}{2}}^{-2}$ in all the diagrams that drops out when we calculate the branching ratio. The remaining dependence on $x_{\frac{3}{2}}$ from the phase space factor is weak. The most significant effect of a large gravitino mass is to move the location of the $\mathrm{Z}$ threshold.

The results for the branching ratios discussed above cannot be directly applied to the 


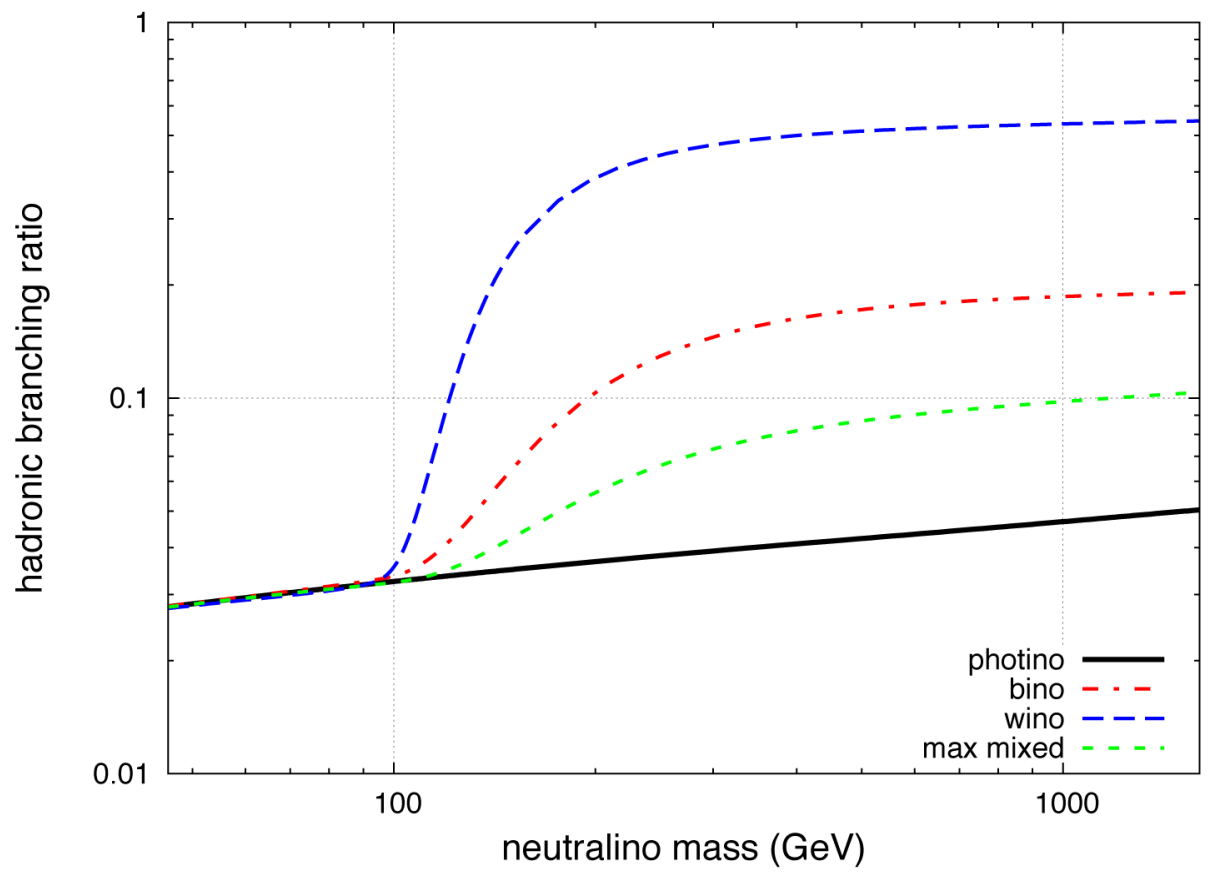

Figure 4. Branching ratio into a quark pair and a gravitino for a Bino or Wino neutralino, for the maximally mixed state and for a photino. Clearly visible is the opening of the $\mathrm{Z}$ channel, which increases the hadronic branching ratio for any state not exactly equal to the photino mixture.

general gaugino or neutralino case, since interference effects between the different diagrams may play a role and either increase the decay rate and/or lower the hadronic branching ratio. Fortunately the main interference effect arises from the neutralino composition. One such case happens for a mixed Bino-Wino state. In this case the branching ratio into quarks is lower than in either of the pure channels. This happens if the Wino-Bino mixture is such to as give an approximately photino NLSP. This significantly reduces the contribution of the $\mathrm{Z}$ channel to the total decay rate whilst boosting the two-body decay into photon and gravitino. This effect can keep the hadronic branching ratio small even above the $\mathrm{Z}$ threshold as shown in figure 4 .

\subsection{Higgsino hadronic branching ratios}

The Higgsino hadronic decays proceed mainly via the intermediate off- or on-shell $\mathrm{Z}$ and Higgs bosons. Below the Higgs threshold the $\mathrm{Z}$ channel is dominant and determines the branching ratio to the different quark flavours. Above the light Higgs threshold the decay into $b \bar{b}$ quarks quickly takes over as it is the dominant Higgs decay channel. In fact in the MSSM the light Higgs mass is always below the threshold for decay into WW and for the heavy Higgses we will consider mostly the case of near-decoupling, where they cannot be produced on-shell in neutralino decays and their contribution to the hadronic branching ratio is completely negligible.

Above the threshold for WW production and below the $t \bar{t}$ threshold from Higgs decay, a 5-body hadronic channel opens up for the Higgsino. In this case we can consider the 

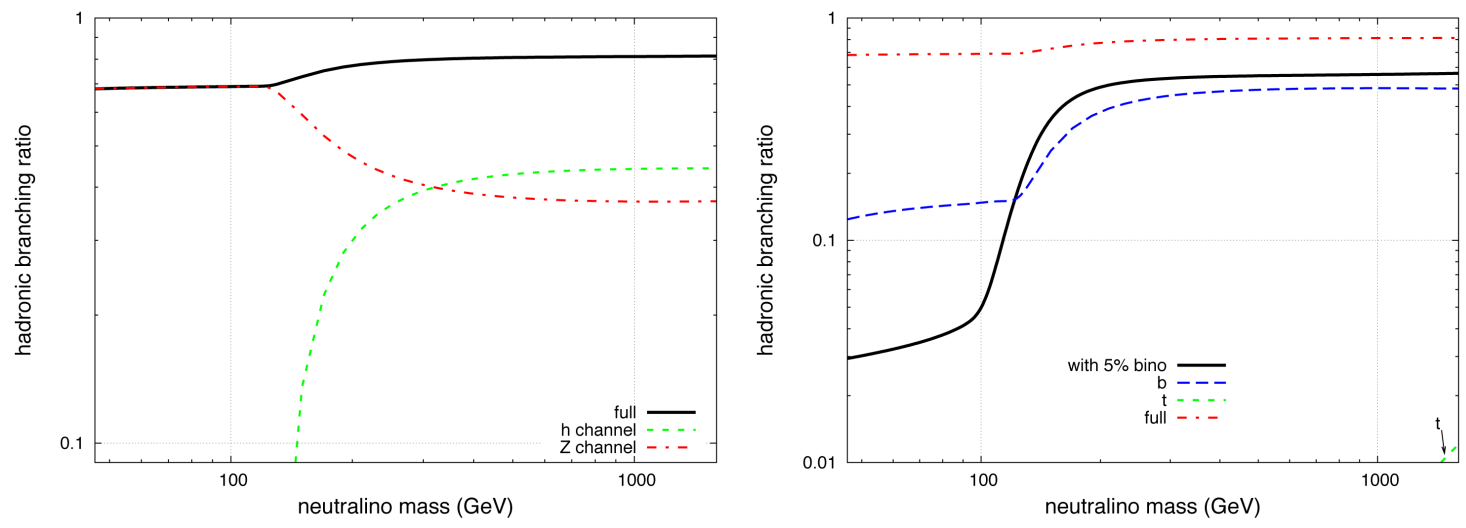

Figure 5. Left Panel: Branching ratio into a quark pair and a gravitino for a Higgsino neutralino in the limit of heavy Higgs decoupling and a SM-like light Higgs. We have taken here $m_{h}=115 \mathrm{GeV}$ and negligible gravitino mass in the phase space factor. Right Panel: As the left panel, but showing the $b \bar{b}$ and $t \bar{t}$ channels and the total hadronic branching ratio with a $5 \%$ Bino fraction. Below the opening of the intermediate Z/Higgs channel, a small Bino fraction can reduce the hadronic branching ratio very effectively.

(heavy) Higgs as on-shell and estimate the contribution from the 5 body-decay using the expression

$$
B_{\text {had }} \sim B_{\text {had }}^{3-b o d y}+\frac{\Gamma\left(\tilde{H} \rightarrow \psi_{3 / 2} H\right)}{\Gamma_{\text {tot }}}\left(1-\left(B_{\text {lep }}^{W}\right)^{2}\right) .
$$

where we have taken the branching ratio of a heavy Higgs into a WW pair to be one. We have checked that this additional channel does not substantially change the Higgsino hadronic branching ratio since it is already quite large at around $80 \%$.

While the hadronic branching ratio for on-shell production is well approximated using the $\mathrm{Z}$ or $h$ hadronic widths, below these thresholds the expressions are more involved and there the 3-body decays are the dominant decay channels for a pure Higgsino. Clearly even in that limit the branching ratio into quarks is large, as shown in the left Panel of figure 5. The expression for the decay into a b quark pair and a gravitino is given, in the limit of negligible final state masses in the phase space and intermediate boson momenta in the propagators, by

$$
\begin{aligned}
\Gamma\left(\tilde{H} \rightarrow \psi_{3 / 2} b \bar{b}\right)= & \frac{\left|-N_{13} \cos \beta+N_{14} \sin \beta\right|^{2}}{48(4 \pi)^{3} M_{P}^{2}} \frac{m_{\tilde{H}}^{5}}{x_{\frac{3}{2}}^{2}} \frac{g_{Z}^{2}}{M_{Z}^{2}}\left[\left(\frac{1}{4}-\frac{2}{3} \sin ^{2} \theta_{W}+\frac{8}{9} \sin ^{4} \theta_{W}\right)\right. \\
& \left.+\frac{3}{10} \frac{\cos ^{2} \alpha}{\sin ^{2} \beta} \frac{\left|-N_{13} \sin \alpha+N_{14} \cos \alpha\right|^{2}}{\left|-N_{13} \cos \beta+N_{14} \sin \beta\right|^{2}} \frac{m_{b}^{2} m_{\tilde{H}}^{2}}{m_{h}^{4}}\right]
\end{aligned}
$$

where the first term comes from the intermediate $\mathrm{Z}$ and the second from the light Higgs. Note that there are no favourable interference effects, so the Higgsino always has a large hadronic branching ratio. On the other hand the presence of even a very small gaugino 
fraction $\sim 0.01-0.05$ already makes the electromagnetic two body decay dominant, effectively suppressing the relative size of the hadronic decays. This effect can be seen in the right panel of figure 5 .

Since in the Higgsino case all processes with intermediate squarks are additionally suppressed by Yukawa couplings compared to the gaugino case, these are surely negligible as soon as the $\mathrm{Z}$ or $\mathrm{h}$ channel opens up. Below these thresholds, squarks that are nearly mass degenerate with a pure Higgsino would further increase its hadronic branching ratio, but again for mass degeneracies such that also coannihilation effects become important.

We summarise the results of this section in the following way: the neutralino usually has a large hadronic branching ratio above the threshold for $\mathrm{Z}$ production. The only way to keep the hadronic branching ratio low for large masses is to engineer a scenario with a photino neutralino. Then the hadronic branching ratio remains below 5-6\% up to $2 \mathrm{TeV}$ masses. We will explore in the following if this may help in relaxing the BBN constraints.

\section{Neutralino thermal abundance and BBN}

The neutralino is considered to be one of the most promising Dark Matter candidates since it is an explicit realisation of the WIMP mechanism. It was observed long ago [22] that a particle with weak interactions and mass at the electroweak scale decouples from the thermal bath with an energy density near to the critical density. In general this density is approximately given by the thermally averaged annihilation cross-section as

$$
\Omega_{\mathrm{WIMP}} h^{2} \simeq \frac{1 \mathrm{pb}}{\langle\sigma v\rangle} .
$$

While this coincidence between the weak scale and DM density is very intriguing and suggestive, realising explicit scenarios with a neutralino WIMP has become increasingly difficult, especially since the accelerator bounds have now pushed the supersymmetric spectrum higher and higher. Nowadays we know that there is much more to the neutralino number density than the approximate formula above and very detailed computations and numerical programs have been developed to take into account all annihilation channels and give reliable predictions in the more sensitive regions such as those near resonances or where there are coannihilations with slightly heavier states. In our analysis we will use one of these numerical packages, Micromegas 2.2 [23], to compute the NLSP number density before decay.

The key quantities in the computation of the thermal abundance are the neutralino composition in gaugino/Higgsino eigenstates and the mass spectrum of the heavier superpartners. To keep our analysis as general as possible we will not fix all supersymmetric parameters according to a specific scenario, but instead we set the soft SUSY breaking parameters at the low energy scale. We keep the majority of the parameters fixed and vary the gaugino and Higgsino soft mass parameters to study how the lifetime and number density vary with the mass and composition of the lightest neutralino. We use Softsusy $2.0[24]$ to compute the physical mass spectrum from the soft SUSY breaking parameters. We have implemented a full numerical computation of the lifetime calculation [18] and 


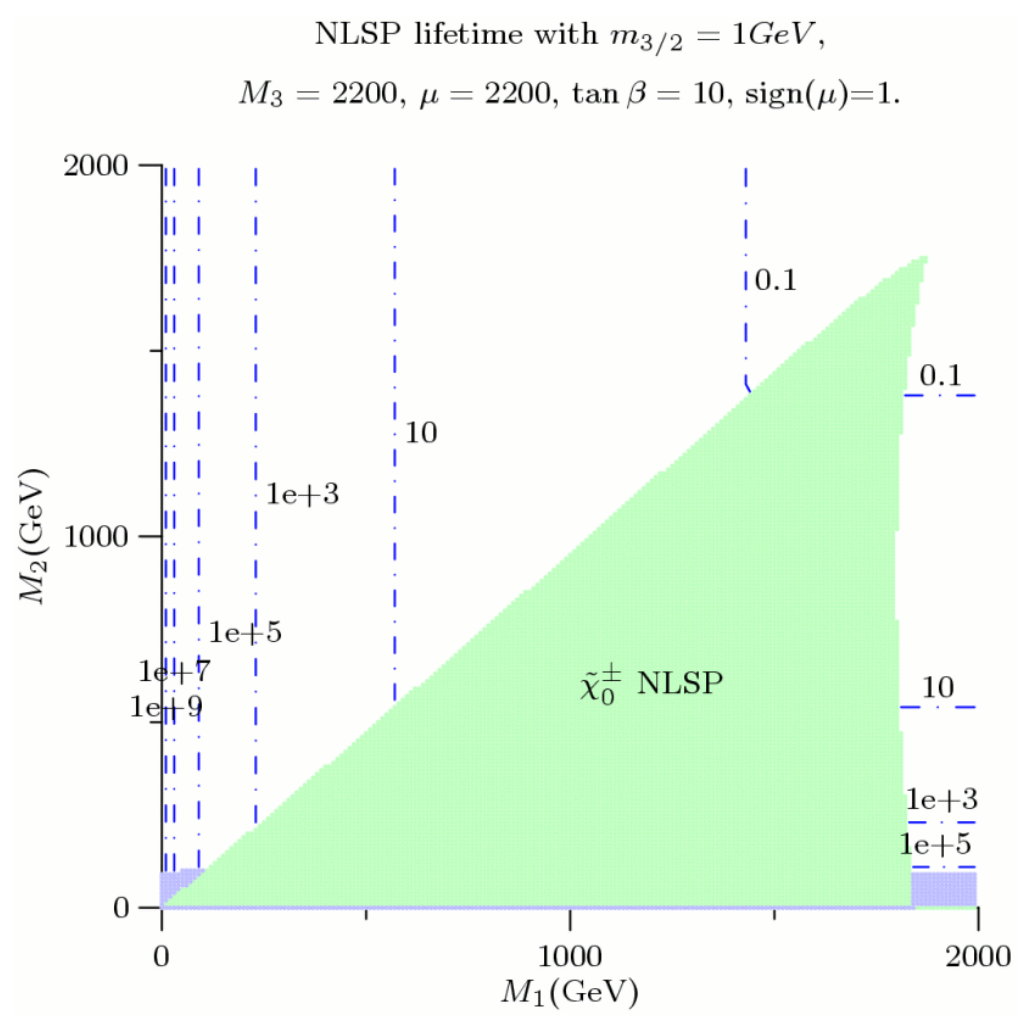

Figure 6. Parameter space of a Bino-Wino neutralino in the $M_{1}, M_{2}$ plane. $M_{1,2}$ refer here to the EW scale soft SUSY breaking gaugino masses. The violet (mid grey) region is excluded by LEP searches for the chargino, while the green (light grey) region has a chargino NLSP. This is because a pure Wino chargino will be lighter than a Wino neutralino. Bino mixing in the neutralino or Higgsino mixing in the chargino is required for the neutralino to be lighter than the chargino. A chargino NLSP is not ruled out physically, but we do not consider it here as we are interested instead in a neutralino NLSP. In the remaining parameter space we calculate the lifetime of the neutralino NLSP for a gravitino mass of $1 \mathrm{GeV}$. The dot-dashed contours show the variation in the lifetime in seconds over the parameter space.

have interfaced this with the SUSY spectrum generated by Softsusy and Micromegas. This allows us to take the results we discussed in the previous section and apply them to general MSSM spectra to see how they fare against the BBN bounds.

\subsection{Bino-Wino NLSP}

We first consider the scenario of a mixed Bino-Wino LSP. To study this scenario we set $\mu$ to be much larger than $M_{1}, M_{2}$, effectively decoupling the Higgsinos from the gauginos. We fix the masses of the sfermions to be above $2 \mathrm{TeV}$ and then vary $M_{1}$ and $M_{2}$ between 0 to $2 \mathrm{TeV}$. The result of this parameter scan is shown in figure 6 .

The mixing changes continuously from practically pure Bino in the left top corner to an equal mixture of Bino-Wino along the diagonal with $M_{1}=M_{2}$ to nearly pure Wino in the bottom right corner. Note though that a small component of Higgsino is always present since we consider a finite value for $\mu$. The lifetime of the neutralino in seconds for 
a gravitino mass of $1 \mathrm{GeV}$ is given by the dot-dashed contours in figure 6 ; it is set by the physical neutralino mass and therefore the contours run parallel to the smaller parameter between $M_{1}$ and $M_{2}$.

We take all the points that satisfied our conditions, i.e. all those with a neutralino NLSP that are not ruled out by LEP bounds, and we calculate the decay lifetime and hadronic branching fraction. We plot these points against the hadronic and electromagnetic BBN bounds in figure 7 . The bounds are taken from the analysis of [16] and the different curves are explained in the figure caption. The vertical axis corresponds to the fraction of the number density that decays to electromagnetic or hadronic products, which we approximate as the number density of the NLSP after freeze-out multiplied by the appropriate branching fraction.

As we can see from figure 7 the hadronic bounds are generally more constraining, but the electromagnetic bounds remain important. This is especially true for light NLSP masses that, as we have discussed, result in a low branching fraction to hadrons. A very light dominantly Wino NLSP almost evades the hadronic bounds for any gravitino mass thanks to the low density and low hadronic branching ratio, but does not overcome the electromagnetic bounds for gravitino masses of $10 \mathrm{GeV}$. The situation is better for smaller gravitino masses and indeed for $m_{\frac{3}{2}}=1 \mathrm{GeV}$ there is a small window for a very light Wino right on the threshold of the LEP bounds that is still allowed even though it has a lifetime greater than $10^{5} \mathrm{~s}$.

Another important feature visible in figure 7 is the large dip that corresponds to resonant annihilation into the pseudo-scalar Higgs, which happens for our choice of parameters for $m_{\tilde{\chi}} \sim 1150 \mathrm{GeV}$. This channel is actually open only thanks to a very small Higgsino component in our gaugino neutralino of less than $0.1 \%$. Nevertheless in the resonant region the annihilation is very efficient, and so it dominates over all Bino annihilation channels. Still it is not enough to avoid the constraints for large lifetimes. The pseudo-scalar Higgs boson has quite a large width and cannot enhance the annihilation by more than a factor approximately given by $\Gamma_{A} / M_{A}$. The light Higgs resonance is more effective, but it is not visible in a plot with this resolution. Also since we chose a Higgs mass of $115 \mathrm{GeV}$, the light Higgs resonance requires a neutralino mass around $57 \mathrm{GeV}$, which is excluded for Wino (and Higgsino) neutralinos thanks to the LEP chargino bound at around $100 \mathrm{GeV}$ [25]. Such a light Bino NLSP will always have a large lifetime for these values of the gravitino mass and will be ruled out by the BBN bounds.

For a gravitino mass of $1 \mathrm{GeV}$ we see that a nearly pure Bino is excluded up to the Higgs resonance at $M_{1}=1150 \mathrm{GeV}$. Conversely a dominantly Wino NLSP avoids the most stringent hadronic BBN bounds through its small number density. This places a lower bound on the allowed Wino mass of $300 \mathrm{GeV}$ for a $1 \mathrm{GeV}$ gravitino, apart for the aforementioned small window right at the LEP mass bound. On the other hand for a $10 \mathrm{GeV}$ gravitino mass, the only allowed cases are either the resonantly annihilating Bino via a small Higgsino component or a Wino in the mass window $800-2000 \mathrm{GeV}$ in the corner between Helium and Deuterium bounds corresponding to a lifetime of around $100 \mathrm{~s}$. Note that for large neutralino masses only the weaker hadronic constraints apply. The bounds are weaker for heavier particles since for constant energy density their number density is 


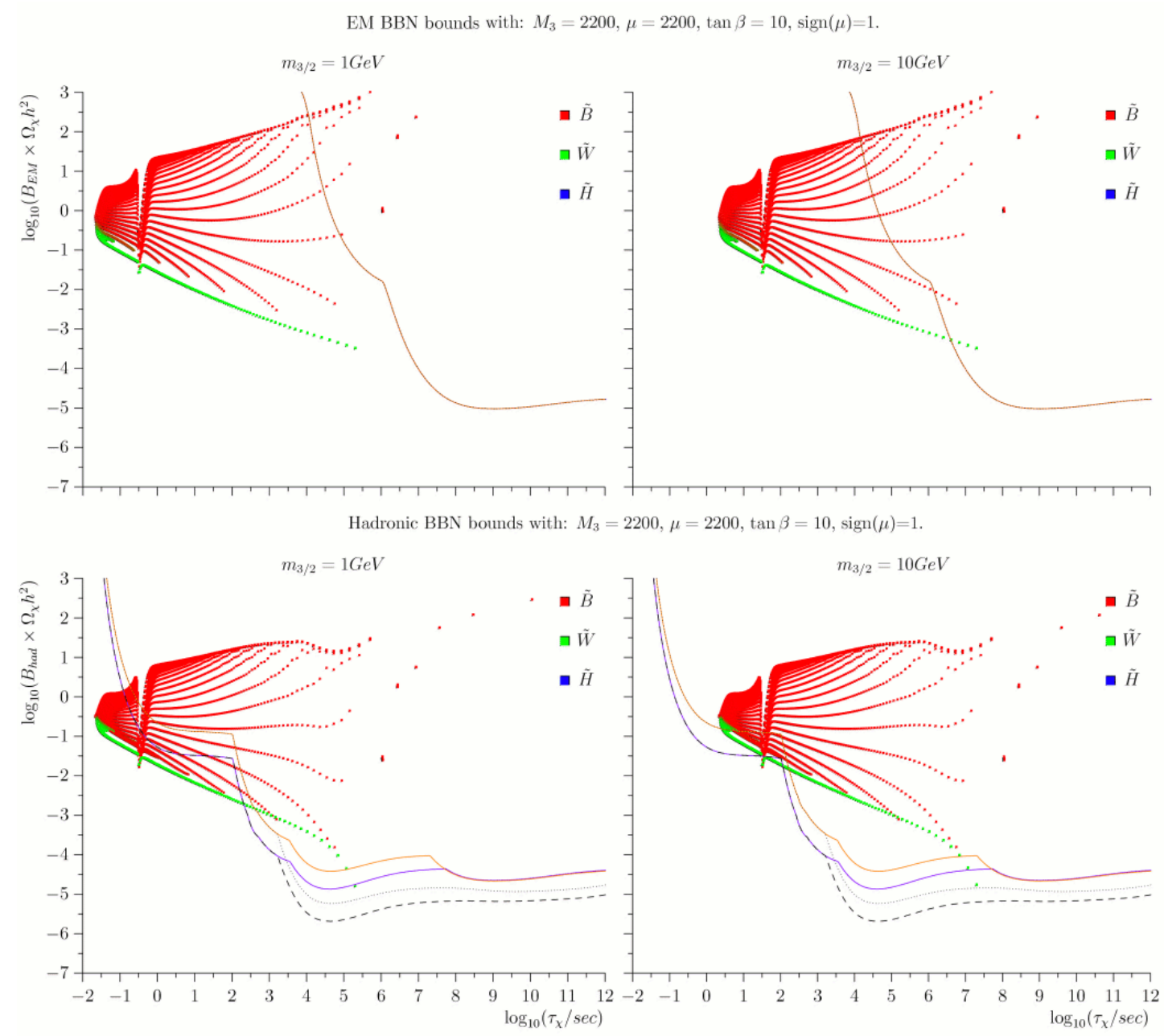

Figure 7. Energy density of the Bino-Wino neutralino compared with the BBN electromagnetic (top) and hadronic constraints (bottom) for the case of a 1 (left) and 10 (right) GeV gravitino mass. The bounds are taken from [16]: the continuous (dashed) lines correspond to more (less) conservative bounds for the ${ }^{6} \mathrm{Li}$ to ${ }^{7} \mathrm{Li}$ ratio, and the region between the curves should not be considered as strictly excluded. The red/upper and violet/lower curves in the hadronic plots are the constraints for $1 \mathrm{TeV}$ and $100 \mathrm{GeV}$ decaying particle mass respectively. The points correspond to the allowed region from figure 6 ; the constant lifetime contours in that figure serve as orientation to infer the neutralino mass and the mass parameters for the present points. The mass increases from right to left as heavier particles decay faster. The composition goes from Bino at the top to Wino at the bottom, and the colours give the dominant component. Note also that the gap between the Bino and Wino neutralino points is due to the presence of the region with a chargino NLSP shown in the figure 6 . The deformation between the upper and lower panels is due to the mass dependence of the hadronic branching ratio with lighter NLSPs having lower branching fractions to hadrons. In contrast the electromagnetic branching ratio is always nearly one.

reduced by the inverse of their mass, while the effect of the hadronic showers grows more slowly than the energy released in the decay.

For larger gravitino masses the points move to the right and more parameter space 


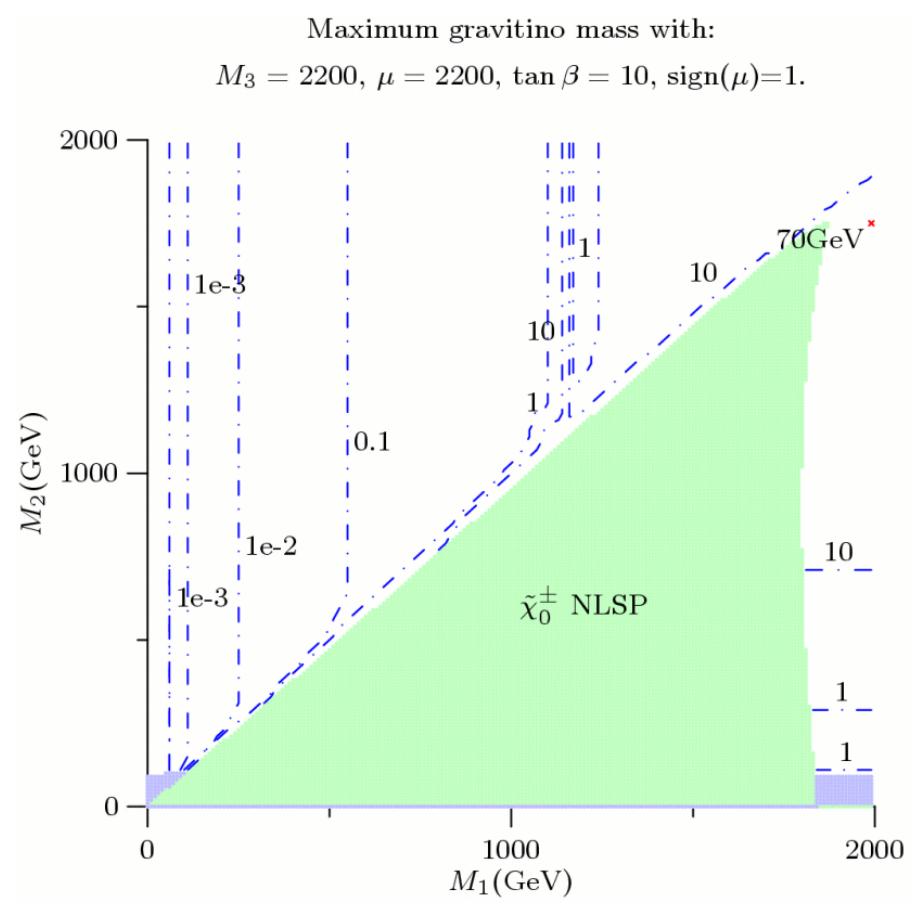

Figure 8. Contours of maximal gravitino mass in $\mathrm{GeV}$ in the $M_{2}$ vs $M_{1}$ plane. The maximal value of $70 \mathrm{GeV}$ is reached at the boundary of our parameter space for a heavy Wino neutralino of about $2 \mathrm{TeV}$ mass, but values around and slightly above $10 \mathrm{GeV}$ are possible also at the Higgs resonance region for a neutralino mass of $m_{\tilde{\chi}} \sim 1150 \mathrm{GeV}$, independently of the Wino-Bino composition, or along the upper edge of the chargino NLSP region where coannihilation helps reduce $\Omega_{N L S P} h^{2}$. Also on the right side there is a window with light Wino, allowing gravitino masses around $1 \mathrm{GeV}$.

becomes excluded. In figure 8 we take each point in the parameter space and find the largest allowed gravitino mass for the given gaugino mass. We find the maximal gravitino mass for a gaugino neutralino below $2 \mathrm{TeV}$ in mass to be $70 \mathrm{GeV}$ and this occurs for a heavy Wino with a small but non-zero Bino fraction. We also see that a Wino NLSP can allow a gravitino of a few $\mathrm{GeV}$ for an NLSP mass of a few hundred $\mathrm{GeV}$, or tens of $\mathrm{GeV}$ for an NLSP with a mass of over $750 \mathrm{GeV}$. On the right of the chargino NLSP region, we find the very light Wino, on the very edge of the LEP bound, which still allows for a gravitino with a mass of a few $\mathrm{GeV}$.

The Bino case is substantially more restrictive. The only regions that allow for a gravitino with a mass over a $\mathrm{GeV}$ are in the regions with a mixed Bino-Wino (along the diagonal in the $\left(M_{1}, M_{2}\right)$ plane), in the pseudo-scalar Higgs resonance which is here around $M_{1}=1150 \mathrm{GeV}$, or for very large Bino masses. In the majority of cases, the rise in the maximum gravitino mass is due to a reduction in the NLSP number density after freeze-out through enhanced annihilation either through the Higgs resonance or along the chargino NLSP region where coannihilation is effective. The reduction of the hadronic branching fraction, e.g. for the fine-tuned photino case, is not sufficient to relax the constraints; the only exception of this is the light Wino case on the LEP boundary, which has both a reduced number density thanks to the strong Wino annihilation and a small hadronic branching ratio around the threshold for intermediate on-shell $\mathrm{Z}$. 


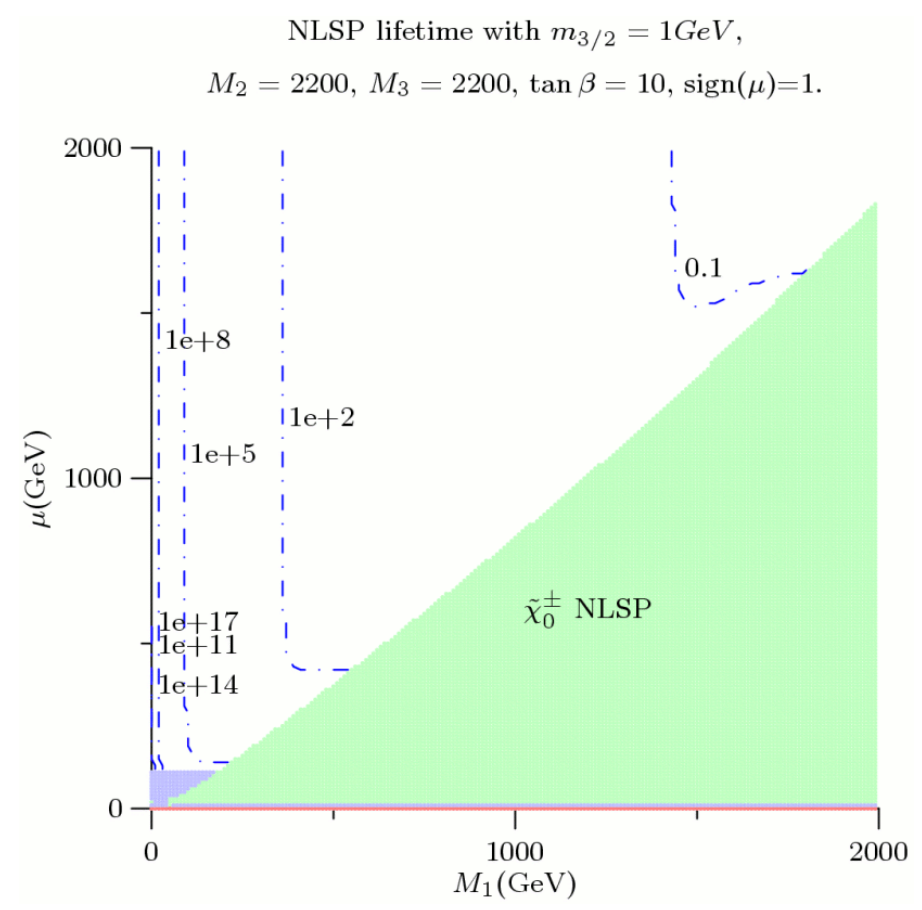

Figure 9. Parameter space of a Bino-Higgsino neutralino. The violet (mid grey) region is excluded by LEP searches, while the green (light grey) region has chargino NLSP. The contours give the neutralino lifetime in seconds for a gravitino mass of $1 \mathrm{GeV}$. The lifetime for other values of the mass is just rescaled as $\left(m_{\frac{3}{2}} / 1 \mathrm{GeV}\right)^{2}$ as long as phase space factors are negligible.

\subsection{Bino-Higgsino NLSP}

In order to study the Bino-Higgsino case we consider a scenario with large $M_{2}$ parameter and study the $\mu, M_{1}$ plane as shown in figure 9 . The other supersymmetric scalar particles are heavy at around $2.2 \mathrm{TeV}$. Note that in this case nearly all the parameter space with $\mu<$ $M_{1}$ corresponds to a Higgsino chargino NLSP and will not be considered here. Therefore we never have a pure Higgsino NLSP and the region with mostly Higgsino composition along the diagonal is characterised by a nearly mass degenerate Higgsino chargino.

The Nucleosynthesis constraints for a mixed Bino-Higgsino case are shown in figure 10. Each point corresponds to a point from figure 9 that satisfies the LEP bounds and give a neutralino NLSP. We see here again that a light mostly Higgsino neutralino, as the Wino, has a suppressed hadronic branching ratio and can nearly evade the hadronic constraints, but not the electromagnetic bounds. In this case the resonant annihilation, both via the pseudo-scalar and via the heavy scalar Higgs, nearly mass degenerate for our choice of parameters, proceeds much more efficiently thanks to the large Higgsino fraction and is thus more prominent. This resonant annihilation overcomes the BBN constraints up to gravitino masses of the order of $70 \mathrm{GeV}$, as can be seen clearly in figure 11 . In this case the coannihilation with the lightest Higgsino chargino serves to keep the Higgsino number density small along the chargino NLSP boundary and consequently results in a larger maximum gravitino mass. However the largest reduction of the number density 

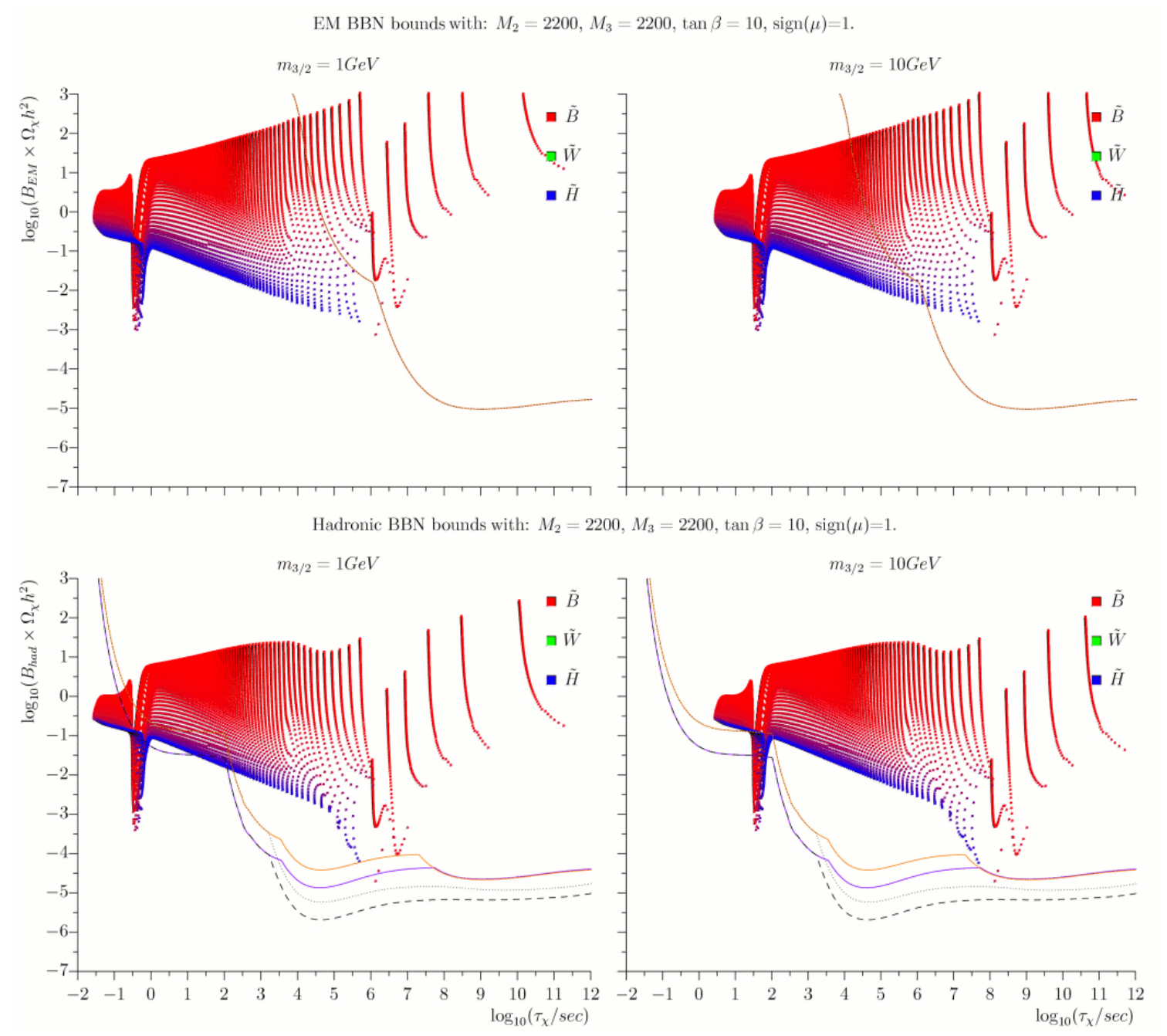

Figure 10. Energy density of the Bino-Higgsino neutralino compared with the BBN electromagnetic (top) and hadronic (bottom) constraints for the case of a 1 (left) and 10 (right) GeV gravitino mass. The bounds are taken from [16]: the continuous (dashed) lines correspond to more (less) conservative bounds for the ${ }^{6} \mathrm{Li}$ to ${ }^{7} \mathrm{Li}$ ratio. The region between these curves should not be considered as strictly excluded, but there a substantial Lithium abundance arises from the NLSP decay. The red/upper and violet/lower curves in the hadronic plots are the constraints for $1 \mathrm{TeV}$ and $100 \mathrm{GeV}$ decaying particle mass respectively. The points correspond to allowed points from figure 9; the constant lifetime contours in that figure serve as orientation to infer the neutralino mass and the mass parameters for the shown points. The neutralino mass increases from right to left and its dominant component is given by the colour coding as indicated. The deformation between the upper and lower panels is due to the mass dependence of the hadronic branching ratio, while the electromagnetic branching ratio is always nearly one.

comes from the heavy Higgs resonance and the rest of the parameter space is excluded for neutralino masses below $2 \mathrm{TeV}$ and gravitino masses above $10 \mathrm{GeV}$. 


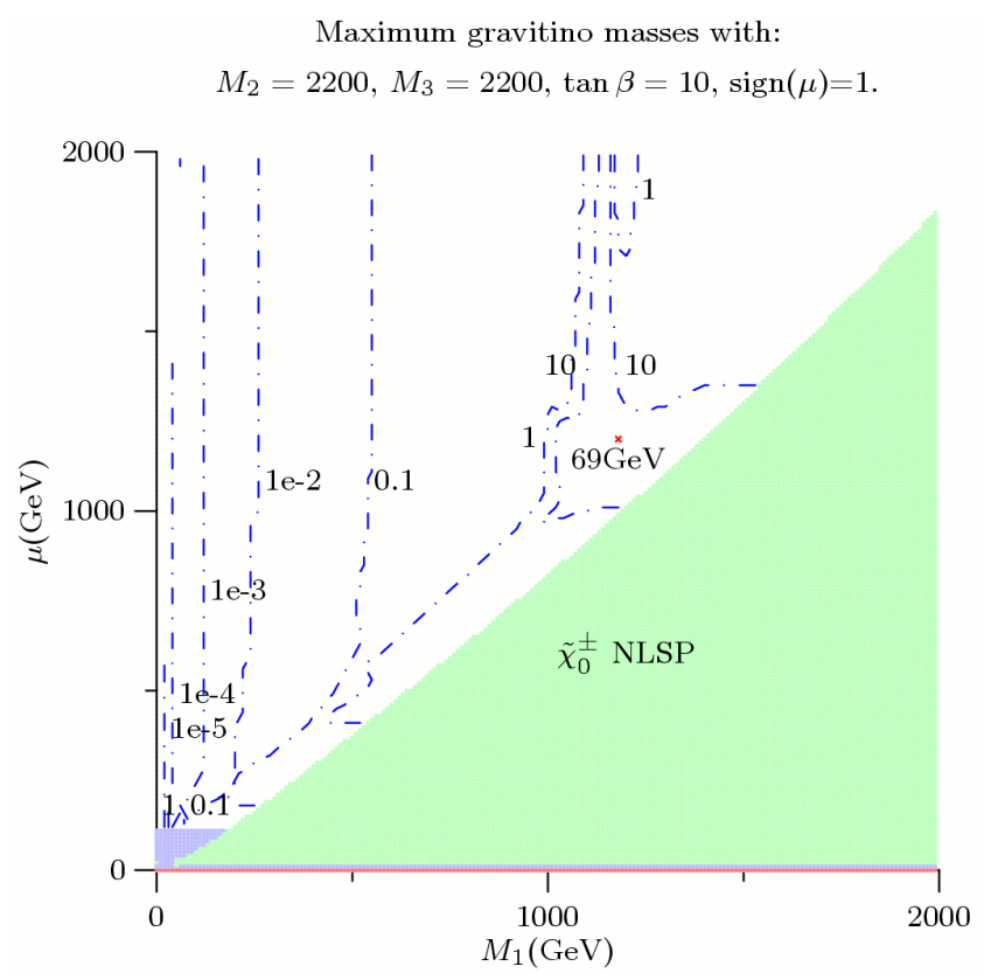

Figure 11. Contours of maximal gravitino mass in $\mathrm{GeV}$ in the $\mu$ vs $M_{1}$ plane. The maximal value of $69 \mathrm{GeV}$ is reached at the heavy Higgs resonance. There is also a thin band at light masses that allows a gravitino of order a few $\mathrm{GeV}$ due to the effect of the light Higgs resonance just above the LEP bound.

\subsection{Wino-Higgsino NLSP}

The Wino-Higgsino case is best considered in the case of a large $M_{1}$ parameter and then the parameter space is given in the $\mu, M_{2}$ plane, as shown in figure 12. The other supersymmetric particles are as heavy as in the previous cases. Also in this case a pure Higgsino is not present in the allowed parameter space, but the Higgsino fraction can be larger than in the Bino-Higgsino case.

The Nucleosynthesis constraints for a mixed Wino-Higgsino case are shown in figure 13. As before, each point corresponds to a point from figure 12 that satisfies LEP bounds and gives a neutralino NLSP. The number density in this case is substantially smaller than for a Bino neutralino, and mostly lower than the DM density for a neutralino mass below $2 \mathrm{TeV}$, but not sufficiently to avoid all the bounds. We see here again that a light Wino-Higgsino has suppressed hadronic branching ratio and can evade all the hadronic constraints, especially if the gravitino mass is sufficiently large to give a suppression of the $\mathrm{Z}$ channel. This effect is most pronounced for $10 \mathrm{GeV}$ gravitino masses since the lightest Wino-Higgsino mass is around $100 \mathrm{GeV}$. However in the case of a $10 \mathrm{GeV}$ gravitino, the light neutralino regions of parameter space are excluded again by the electromagnetic constraints. As in the Wino-Bino case, we see here that there is a small window for a Wino with a mass just above the LEP bound and a gravitino of around $1 \mathrm{GeV}$. However in 


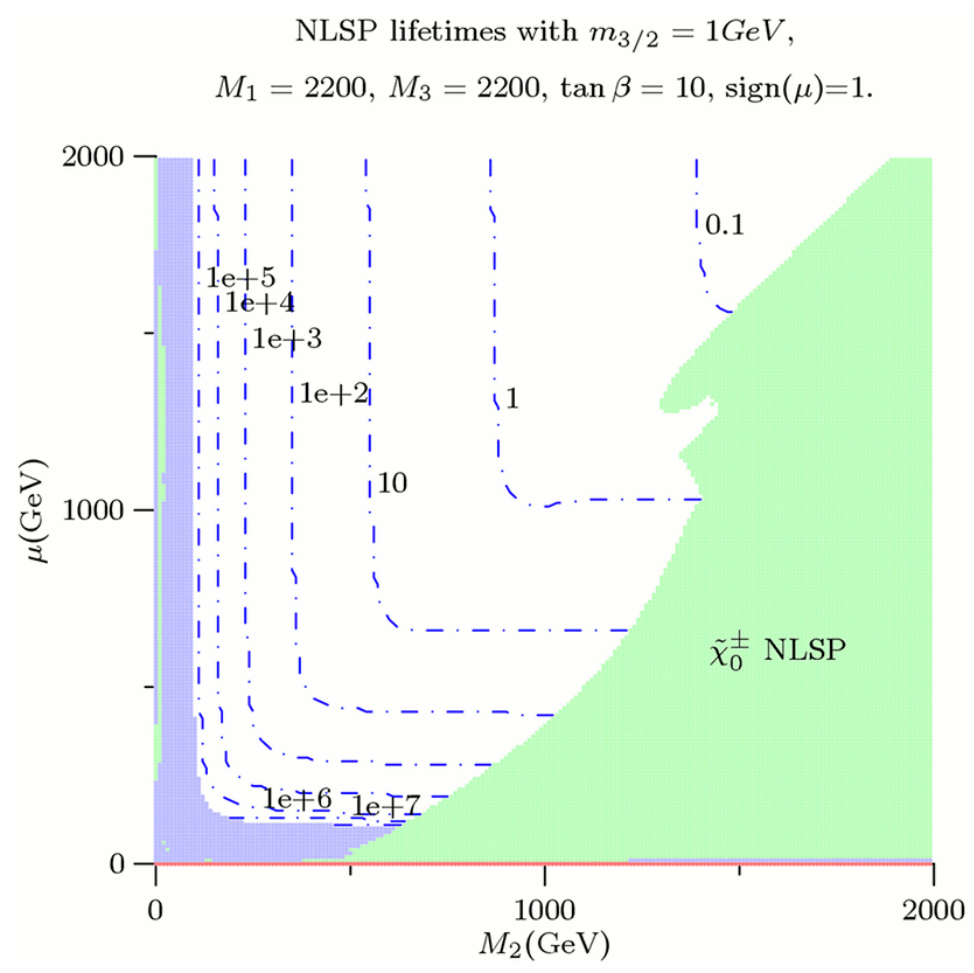

Figure 12. Parameter space of a Wino-Higgsino neutralino. The violet (mid grey) region is excluded by LEP searches, while the green (light grey) region has a chargino NLSP. The contours give the neutralino lifetime in seconds for a gravitino mass of $1 \mathrm{GeV}$. The lifetime for other values of $m_{\frac{3}{2}}$ is just rescaled as $\left(m_{\frac{3}{2}} / 1 \mathrm{GeV}\right)^{2}$ as long as phase space factors are negligible.

most cases in the low mass region, below the $\mathrm{Z}$ resonance, the interplay of the two types of bounds exclude a light neutralino NLSP.

In this case, for gravitino masses of $10 \mathrm{GeV}$, apart from the resonant annihilation case at $1150 \mathrm{GeV}$ neutralino mass, once again there is a surviving window for an NLSP mass of 800-2000 GeV in the corner between Helium and Deuterium bounds. This window remains open up to gravitino masses of about $70 \mathrm{GeV}$. It is not surprising that we find a very similar maximal value for all our cases, since in two of them it is located at the boundary of our parameter region, where the neutralino is actually a fully mixed state. Note however that in the case of a Wino-Higgsino neutralino there is a wide region of allowed gravitino masses between 10 and $69 \mathrm{GeV}$, for neutralino masses above $800 \mathrm{GeV}$, which is not limited to the Higgs resonance. This is shown in figure 14. So we can conclude that the BBN constraints are strongly relaxed for the Wino-Higgsino case thanks to the low relic density.

\section{General neutralino NLSP}

Combining the results of the previous sections we can obtain the general result for any neutralino composition. We observe the following features: 
EM BBN bounds with: $M_{1}=2200, M_{3}=2200, \tan \beta=10, \operatorname{sign}(\mu)=1$.

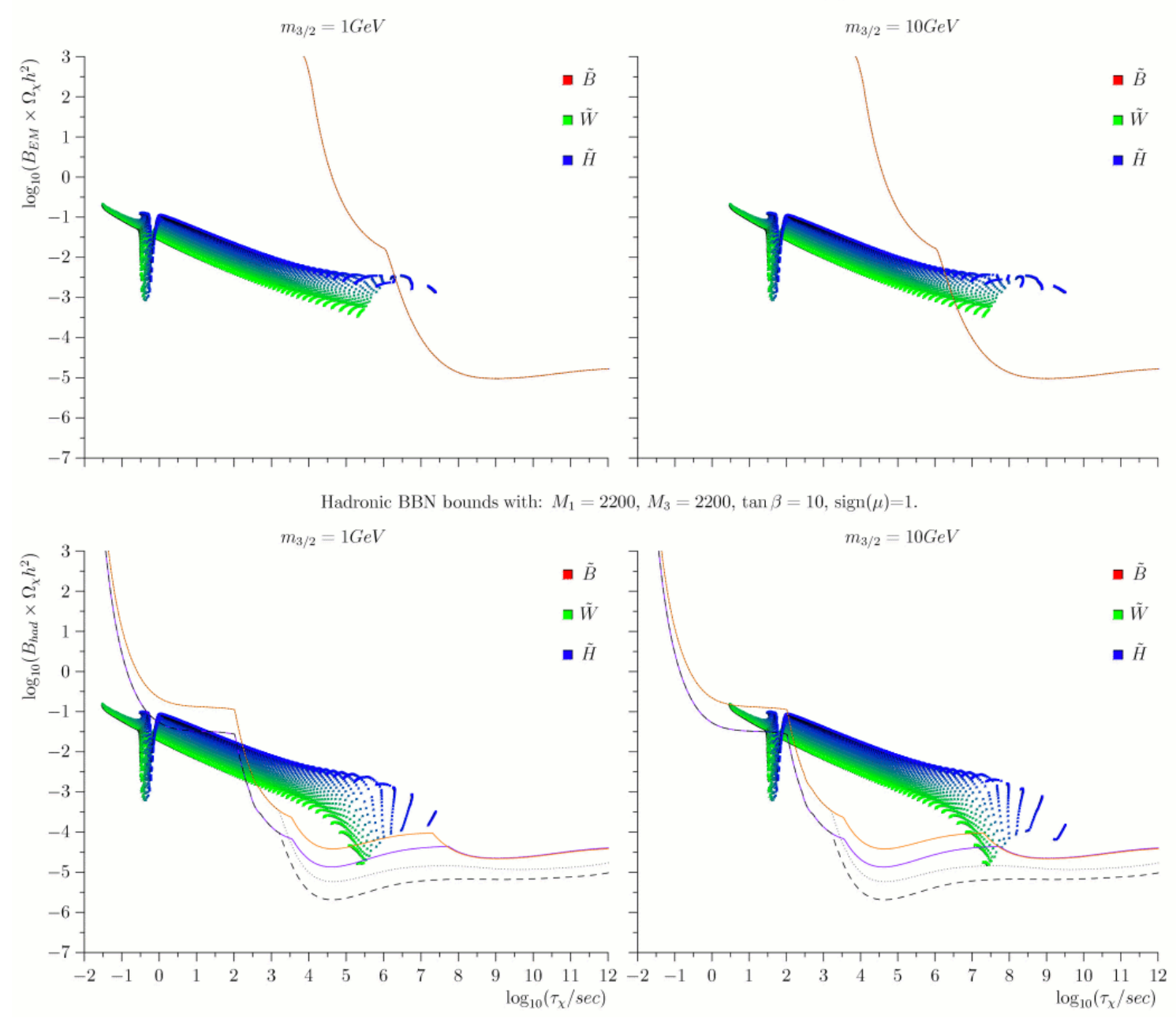

Figure 13. Energy density for a Wino-Higgsino neutralino compared with the BBN electromagnetic (top) and hadronic (bottom) constraints for the case of a 1 (left) and 10 (right) GeV gravitino mass. The bounds are taken from [16]: the continuos (dashed) lines correspond to more (less) conservative bounds for the ${ }^{6} \mathrm{Li}$ to ${ }^{7} \mathrm{Li}$ ratio, and the region between these curves should not be considered as strictly excluded, but there a substantial Lithium abundance arises from the NLSP decay. The red/upper and violet/lower curves in the hadronic plots are the constraints for $1 \mathrm{TeV}$ and $100 \mathrm{GeV}$ decaying particle mass respectively. The points correspond to allowed points from figure 12; again the constant lifetime contours in that figure serve as orientation to infer the neutralino mass and the mass parameters for the present points. The NLSP mass increases from right to left and the dominant component is given by the colour coding. The deformation between the upper and lower panels is due to the mass dependence of the hadronic branching ratio, while the electromagnetic branching fraction is always nearly one.

- The reduction of the hadronic branching ratio is more effective for low neutralino masses, thanks to the phase space suppression for the $\mathrm{Z}$ and Higgs channels, and can help to overcome the BBN bounds for Wino or Higgsino neutralinos. Unfortunately in most cases the electromagnetic constraints are not bypassed. This effect does not 


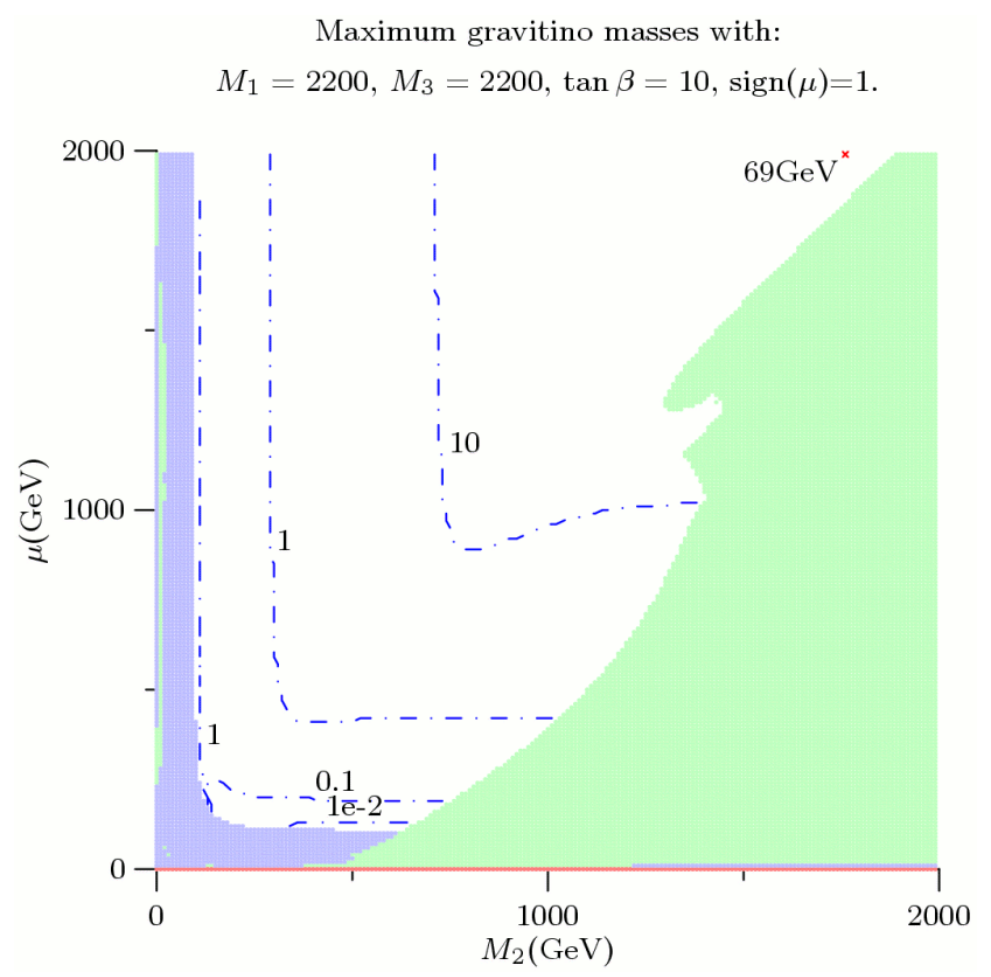

Figure 14. Contours of maximal gravitino mass in $\mathrm{GeV}$ in the $\mu$ vs $M_{2}$ plane. The maximal value of $69 \mathrm{GeV}$ is reached again at the boundary of the parameter space for a neutralino mass of about $2 \mathrm{TeV}$. We also see a thin band at $M_{2} \approx 100 \mathrm{GeV}$ where the low hadronic branching fraction allows a gravitino mass up to a few $\mathrm{GeV}$.

allow for gravitino masses above few $\mathrm{GeV}$.

- The suppression of the hadronic channels due to a photino composition is not sufficient to relax the hadronic constraints, since the photino relic density is always too large. Moreover for low masses and large lifetimes the photino is excluded by the electromagnetic bounds.

- The resonant Higgs annihilation can lower the relic density sufficiently to allow for gravitino masses up to $70 \mathrm{GeV}$ for our choice of heavy Higgs masses. This maximal value comes from the point at which the resonant spike touches the BBN bounds at around $10^{2.5-3} \mathrm{~s}$ and therefore for the general case the maximal gravitino mass is given approximately by the neutralino mass as

$$
\left(m_{3 / 2}\right)_{\text {max }, \text { res }} \sim 70 \mathrm{GeV}\left(\frac{m_{\tilde{\chi}}}{1.15 \mathrm{TeV}}\right)^{5 / 2} .
$$

The actual value is therefore smaller for lower neutralino and heavy Higgs masses and cannot allow for $10 \mathrm{GeV}$ gravitino mass for neutralino masses below $500 \mathrm{GeV}$.

- The coannihilation with the charginos and the stronger annihilations characteristic of Wino and Higgsino NLSPs result in a lower number density of NLSPs in the early 
universe. This helps evade the strongest BBN bounds and results in larger allowed gravitino masses than for a predominantly Bino NLSP.

- In the previous sections we kept the sfermions heavy and focused on the effect of the neutralino composition. In many cases we would expect the sfermion masses to be close in mass to the lightest neutralino, particularly in cases with a gaugino NLSP and light sleptons. Then sfermion coannihilation can significantly reduce the number density of a neutralino NLSP. We studied the sfermion coannihilation case and found it to have a significant effect on the Bino NLSP number density, allowing an order of magnitude increase in the maximum gravitino mass. Conversely, in the case of a dominantly Higgsino or Wino NLSP, the number density is only slightly altered by sfermion coannihilation and the resulting change in the number density has little impact on the allowed gravitino mass. We show the effect of coannihilation with a light stau for a Bino NLSP in figure 15 and we obtain qualitatively similar results for a stop NNLSP.

- We have also studied the effect of changing the value of $\tan \beta$. This has little impact on the lifetime calculations, instead having the most direct effect through the location of the Higgs resonances for the NLSP annihilations.

We see therefore that a general neutralino NLSP extends the range of gravitino masses allowed by about one order of magnitude compared to the Bino case. Our study shows that the smallest splitting between the allowed gravitino mass and NLSP mass occurs in the regions of resonant annihilation or Wino-Higgsino neutralinos. In these cases a substantial hierarchy remains necessary between the NLSP and the gravitino mass.

\section{Conclusions}

We have considered in this paper the BBN constraints on a general neutralino NLSP for varying gravitino masses. We found that the bounds are weaker for the case of a Wino or Higgsino neutralino, but still not sufficiently relaxed to allow a $100 \mathrm{GeV}$ mass gravitino, unless we allow for neutralino masses in excess of $2 \mathrm{TeV}$. The maximal gravitino mass we find for neutralinos below $2 \mathrm{TeV}$ is around $70 \mathrm{GeV}$, mostly at the boundary of the parameter space. Nevertheless gravitino masses in the 10-70 GeV window are possible, even for lower neutralino masses, especially in regions with resonant annihilation of the NLSP into heavy scalar or pseudo-scalar Higgs or for a Wino-Higgsino neutralino. These values of the gravitino mass may still be marginally in agreement with the thermal leptogenesis scenario [5] if the gluinos are light [26] . For our choice of parameters, i.e. $M_{3}=2.2 \mathrm{TeV}$, they are unfortunately too heavy, but lowering their mass should not modify our results too much, especially in the Higgs resonance region. Then assuming them to be just a bit heavier than the neutralino, and a gravitino mass of $70 \mathrm{GeV}$, the reheat temperature for gravitino Dark Matter is given by

$$
T_{R} \sim 1.5 \times 10^{9} \mathrm{GeV}\left(\frac{m_{\tilde{g}}}{1.25 \mathrm{TeV}}\right)^{-2} .
$$


NLSP Lifetime with $m_{3 / 2}=1 G e V$ : $M_{2}=520, M_{3}=800, \mu=520, \tan \beta=10, \operatorname{sign}(\mu)=1$.

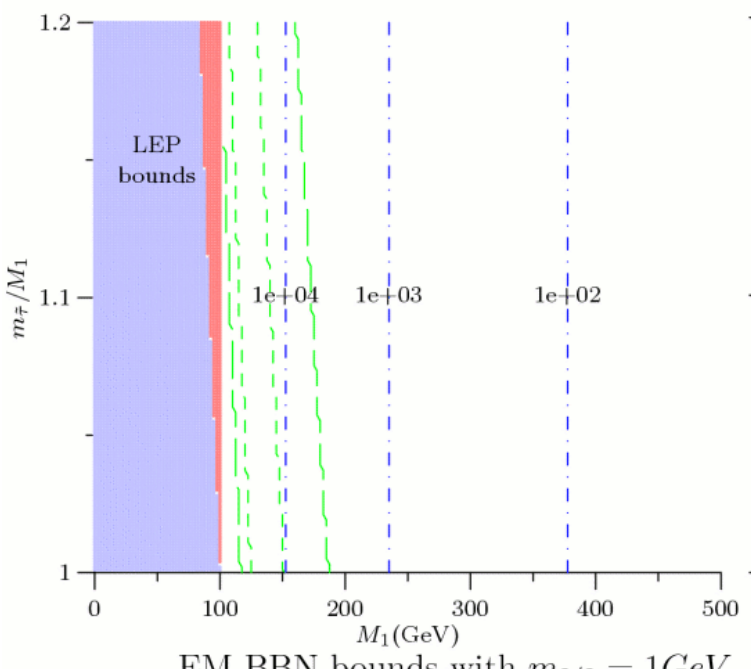

EM BBN bounds with $m_{3 / 2}=1 \mathrm{GeV}$
Maximum gravitino mass with:

$M_{2}=520, M_{3}=800, \mu=520, \tan \beta=10, \operatorname{sign}(\mu)=1$.

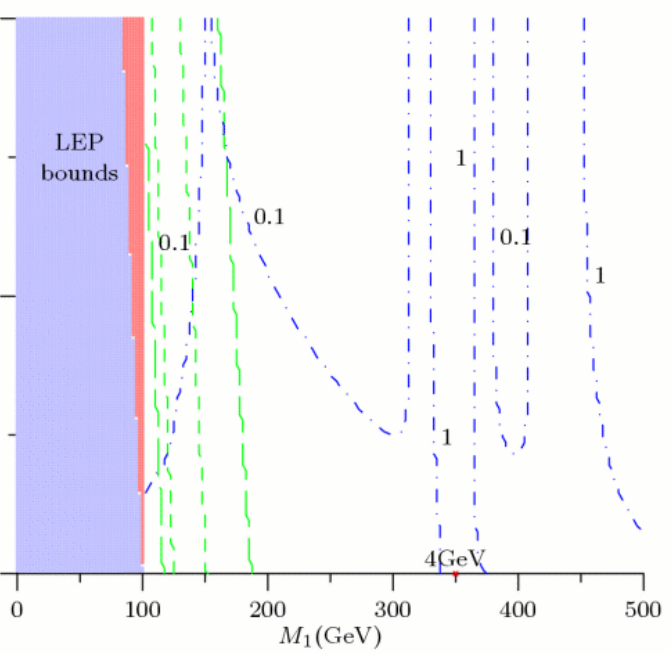

Hadronic BBN bounds with $m_{3 / 2}=1 \mathrm{GeV}$
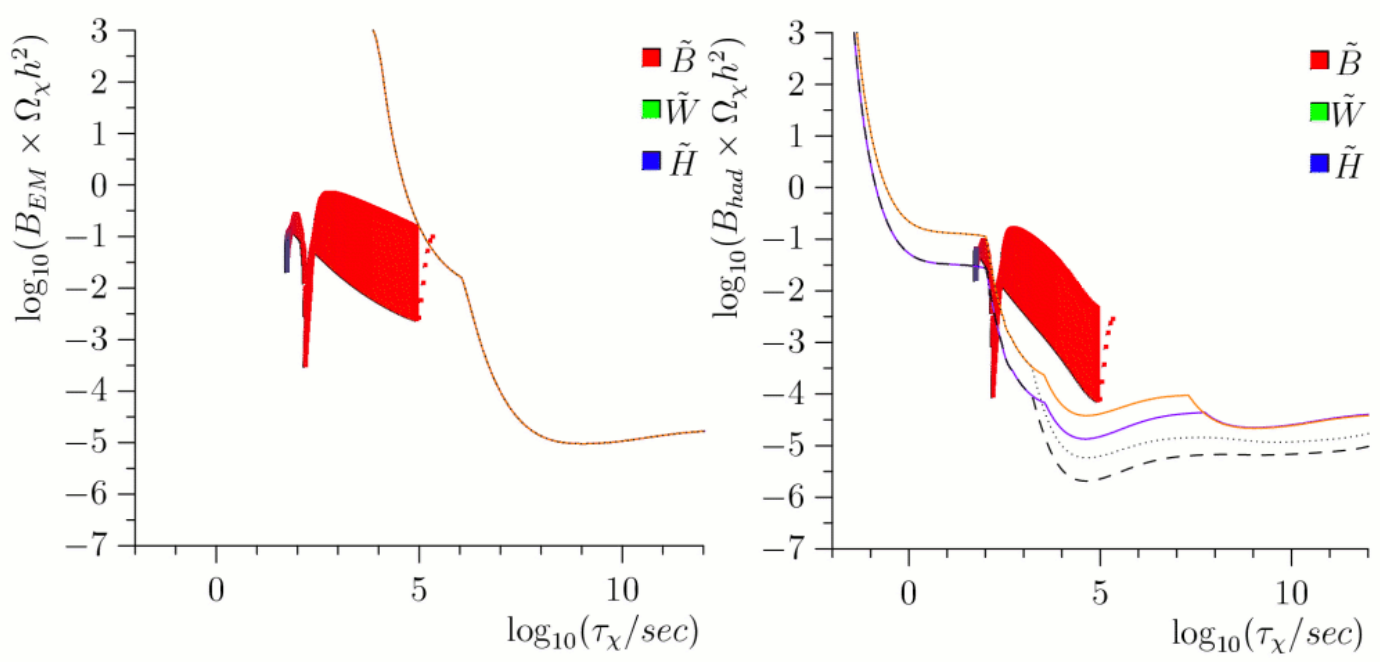

Figure 15. The effect of coannihilation with a light stau for a Bino NLSP. In the top two plots we show the parameter space: on the horizontal axis we take the increasing Bino mass, while the vertical axis shows the mass splitting between the neutralino NLSP and the stau NNLSP. The top left plot shows the lifetime contours for a $1 \mathrm{GeV}$ gravitino in seconds. Note that the lifetime of the neutralino is dependent on the neutralino mass, but insensitive to the stau mass. The top right plot shows the maximum gravitino mass across the parameter space. The overall behaviour is that the maximum mass increases with increasing neutralino mass and shortening lifetime. The exception to this behaviour is around $350 \mathrm{GeV}$ where we hit the Higgs resonance, here we have taken the pseudo-scalar Higgs mass to be $700 \mathrm{GeV}$. We also see an increase in the maximum mass as the mass splitting approaches zero. Here the number density is reduced enough to avoid the ${ }^{4} \mathrm{He}$ bound, corresponding to lifetimes below $100 \mathrm{~s}$. This results in an order of magnitude increase in the maximum allowed gravitino mass.

Since the bound $T_{R}>1.5 \times 10^{9} \mathrm{GeV}$ has been obtained for thermal leptogenesis requiring independence from the initial conditions [27], our scenario may still be acceptable, within 
the order one uncertainty in the thermal computation, especially with a small enhancement of the $\mathrm{CP}$ violation. This low value of the gluino mass would again call for non universal gaugino masses at the GUT scale.

Another open window in the parameter space is the Wino NLSP region just above the LEP bound, where kinematic effects conspire to reduce the neutralino hadronic branching ratio. In that case the gravitino mass is of the order of a few GeVs and the gluino mass may be substantially smaller than $1 \mathrm{TeV}$, such as to still allow for successful leptogenesis.

A general lowering of the $M_{3}$ mass parameter in comparison to the other masses is also welcome in order to observe this scenario at the LHC. Assuming the gluinos to be below $2 \mathrm{TeV}$ of mass, the main observable will be missing energy in the cascade decays, as for the case of neutralino Dark Matter. Unfortunately, the resonant annihilation region is difficult to investigate at the LHC, since very precise measurements of the neutralino and heavy Higgs masses are necessary to disentangle the neutralino LSP and DM case from the one we discuss here. In contrast, a Wino-Higgsino NLSP scenario is more easily identified due to the existence of nearly degenerate charginos. Nevertheless it will probably be difficult to prove that the neutralino number density is much lower that required for dark matter from LHC measurements alone.

Regarding the exploitation of the NLSP decay to produce the whole DM density, it is not possible for a Wino-Higgsino neutralino in our parameter space due to the small relic density, below 0.1. For a Bino NLSP, the ratio between gravitino and neutralino mass is always smaller than $10^{-3}$ away from the Higgs resonance, where the relic density is in any case suppressed. From the figures 7 and 10 we see that even the Bino relic density for a $2 \mathrm{TeV}$ mass is not sufficient to compensate a factor of $10^{-3}$ reduction in the energy density and produce the whole gravitino Dark Matter population. Much heavier NLSP neutralinos are needed to obtain the right abundance and avoid BBN bounds for gravitino masses of $1-10 \mathrm{GeV}[28]$. Such large masses are unfortunately beyond the reach of the LHC and cannot be reconciled with thermal leptogenesis.

From our analysis we can in general conclude that non-universal gaugino masses with a compressed gaugino spectrum, and moreover NLSP masses above $500 \mathrm{GeV}$ or so are preferred. Any evidence of a light neutralino at LHC, apart for the case of a light Wino with nearly degenerate charginos, would be difficult to reconcile with gravitino Dark Matter and leptogenesis in the standard cosmology picture with R-parity conservation. On the other hand, heavier neutralino masses and strong enhancement of the NLSP annihilation as in the Higgs resonance case may be the first phenomenological signal for gravitino DM at colliders.

Note added. During the completion of this work, ref. [29] appeared, which also computes Nucleosynthesis constraints for a general neutral decaying particle and in particular for a decaying gravitino. In [29] no BBN bound for lifetimes below $100 \mathrm{~s}$ are given, due to a weaker upper limit on ${ }^{4} \mathrm{He}$ compared to [16]. Such weakening of the bounds would not have any effect on our results for the Wino-Higgsino case, since there the relic density is below the ${ }^{4}$ He curve, but it may open up more parameter space for the Bino neutralino, beyond the Higgs resonance region. 


\section{Acknowledgments}

LC would like to thank W. Buchmüller, S. Davidson, A. Romanino, M. Serone, F. Strumia and F. Zwirner for useful discussions on the WW decay channel. LC would also like to thank the Aspen Center for Physics for hospitality during the final stages of this work.

LC and JR acknowledge financial support via the "Marie Curie Host Fellowship for Transfer of Knowledge" MTKD-CT-2005-029466 during their stay in the Institute for Theoretical Physics, University of Warsaw, where this work has been started. LC acknowledges the support of the "Impuls- und Vernetzungsfond" of the Helmholtz Association under the contract number VH-NG-006. The work of JR is supported by the NSF Career Grant PHY-0449818 and DOE OJI grant \#DE-FG02-06ER41417.

JH is supported by the Deutsche Forschungsgemeinschaft via the Junior Research Group "SUSY Phenomenology" within the Collaborative Research Centre 676 "Particles, Strings and the Early Universe".

\section{References}

[1] H. Pagels and J.R. Primack, Supersymmetry, Cosmology and New TeV Physics, Phys. Rev. Lett. 48 (1982) 223 [SPIRES].

[2] For a review see G.F. Giudice and R. Rattazzi, Theories with gauge-mediated supersymmetry breaking, Phys. Rept. 322 (1999) 419 [hep-ph/9801271] [SPIRES].

[3] D.E. Kaplan, G.D. Kribs and M. Schmaltz, Supersymmetry breaking through transparent extra dimensions, Phys. Rev. D 62 (2000) 035010 [hep-ph/9911293] [SPIRES];

Z. Chacko, M.A. Luty, A.E. Nelson and E. Ponton, Gaugino mediated supersymmetry breaking, JHEP 01 (2000) 003 [hep-ph/9911323] [SPIRES].

[4] W. Buchmüller, K. Hamaguchi and J. Kersten, The gravitino in gaugino mediation, Phys. Lett. B 632 (2006) 366 [hep-ph/0506105] [SPIRES].

[5] For general reviews see: S. Davidson, E. Nardi and Y. Nir, Leptogenesis, Phys. Rept. 466 (2008) 105 [arXiv: 0802.2962] [SPIRES];

W. Buchmüller, R.D. Peccei and T. Yanagida, Leptogenesis as the origin of matter, Ann. Rev. Nucl. Part. Sci. 55 (2005) 311 [hep-ph/0502169] [SPIRES].

[6] M. Bolz, A. Brandenburg and W. Buchmüller, Thermal Production of Gravitinos, Nucl. Phys. B 606 (2001) 518 [Erratum ibid. B 790 (2008) 336] [hep-ph/0012052] [SPIRES];

J. Pradler and F.D. Steffen, Thermal Gravitino Production and Collider Tests of Leptogenesis, Phys. Rev. D 75 (2007) 023509 [hep-ph/0608344] [SPIRES].

[7] J.R. Ellis, D.V. Nanopoulos and S. Sarkar, The Cosmology of Decaying Gravitinos, Nucl. Phys. B 259 (1985) 175 [SPIRES];

D. Lindley, Cosmological Constraints on the Lifetime of Massive Particles, Astrophys. J. 294 (1985) 1 [SPIRES];

M.H. Reno and D. Seckel, Primordial Nucleosynthesis: The Effects of Injecting Hadrons, Phys. Rev. D 37 (1988) 3441 [SPIRES];

S. Dimopoulos, R. Esmailzadeh, L.J. Hall and G.D. Starkman, Is the universe closed by baryons? Nucleosynthesis with a late decaying massive particle, Astrophys. J. 330 (1988) 545 [SPIRES]; 
R.J. Scherrer and M.S. Turner, Primordial Nucleosynthesis with Decaying Particles. 1. Entropy Producing Decays. 2. Inert Decays, Astrophys. J. 331 (1988) 19 [Astrophys. J. 331 (1988) 33] [SPIRES];

J.R. Ellis, G.B. Gelmini, J.L. Lopez, D.V. Nanopoulos and S. Sarkar, Astrophysical constraints on massive unstable neutral relic particles, Nucl. Phys. B 373 (1992) 399 [SPIRES];

For general reviews see S. Sarkar, Big bang nucleosynthesis and physics beyond the standard model, Rept. Prog. Phys. 59 (1996) 1493 [hep-ph/9602260] [SPIRES];

K. Jedamzik and M. Pospelov, Big Bang Nucleosynthesis and Particle Dark Matter, New J. Phys. 11 (2009) 105028 [arXiv: 0906 . 2087] [SPIRES].

[8] J.R. Ellis, K.A. Olive, Y. Santoso and V.C. Spanos, Gravitino dark matter in the CMSSM, Phys. Lett. B 588 (2004) 7 [hep-ph/0312262] [SPIRES];

L. Roszkowski, R. Ruiz de Austri and K.-Y. Choi, Gravitino dark matter in the CMSSM and implications for leptogenesis and the LHC, JHEP 08 (2005) 080 [hep-ph/0408227] [SPIRES];

J.R. Ellis, K.A. Olive and E. Vangioni, Effects of unstable particles on light-element abundances: Lithium versus deuterium and He-3, Phys. Lett. B 619 (2005) 30 [astro-ph/0503023] [SPIRES];

D.G. Cerdeno, K.-Y. Choi, K. Jedamzik, L. Roszkowski and R. Ruiz de Austri, Gravitino dark matter in the CMSSM with improved constraints from BBN, JCAP 06 (2006) 005 [hep-ph/0509275] [SPIRES];

S. Bailly, K.-Y. Choi, K. Jedamzik and L. Roszkowski, A Re-analysis of Gravitino Dark Matter in the Constrained MSSM, JHEP 05 (2009) 103 [arXiv:0903.3974] [SPIRES].

[9] J.L. Feng, S. Su and F. Takayama, Supergravity with a gravitino LSP, Phys. Rev. D 70 (2004) 075019 [hep-ph/0404231] [SPIRES].

[10] M. Kawasaki, K. Kohri, T. Moroi and A. Yotsuyanagi, Big-Bang Nucleosynthesis and Gravitino, Phys. Rev. D 78 (2008) 065011 [arXiv:0804.3745] [SPIRES].

[11] C. Cheung, A.L. Fitzpatrick and D. Shih, (Extra)Ordinary Gauge Mediation, JHEP 07 (2008) 054 [arXiv: 0710.3585] [SPIRES];

P. Meade, N. Seiberg and D. Shih, General Gauge Mediation, Prog. Theor. Phys. Suppl. 177 (2009) 143 [arXiv:0801.3278] [SPIRES];

L.M. Carpenter, M. Dine, G. Festuccia and J.D. Mason, Implementing General Gauge Mediation, Phys. Rev. D 79 (2009) 035002 [arXiv: 0805. 2944] [SPIRES];

M. Buican, P. Meade, N. Seiberg and D. Shih, Exploring General Gauge Mediation, JHEP 03 (2009) 016 [arXiv: 0812 .3668] [SPIRES].

[12] W. Buchmüller, L. Covi, J. Kersten and K. Schmidt-Hoberg, Dark matter from gaugino mediation, JCAP 11 (2006) 007 [hep-ph/0609142] [SPIRES].

[13] L. Randall and R. Sundrum, Out of this world supersymmetry breaking, Nucl. Phys. B 557 (1999) 79 [hep-th/9810155] [SPIRES];

G.F. Giudice, M.A. Luty, H. Murayama and R. Rattazzi, Gaugino Mass without Singlets, JHEP 12 (1998) 027 [hep-ph/9810442] [SPIRES].

[14] J.R. Ellis, K. Enqvist, D.V. Nanopoulos and K. Tamvakis, Gaugino masses and grand unification, Phys. Lett. B 155 (1985) 381 [SPIRES];

M. Drees, Phenomenological consequences of $N=1$ supergravity theories with nonminimal kinetic energy terms for vector superfields, Phys. Lett. B 158 (1985) 409 [SPIRES].

[15] P. Langacker, G. Paz, L.-T. Wang and I. Yavin, Aspects of Z'-mediated Supersymmetry Breaking, Phys. Rev. D 77 (2008) 085033 [arXiv:0801.3693] [SPIRES]. 
[16] K. Jedamzik, Big bang nucleosynthesis constraints on hadronically and electromagnetically decaying relic neutral particles, Phys. Rev. D 74 (2006) 103509 [hep-ph/0604251] [SPIRES].

[17] S.P. Martin, A Supersymmetry Primer, hep-ph/9709356 [SPIRES].

[18] For a review of the gravitino couplings, the Feynman rules used here and the details of the computation, see J. Hasenkamp, General Neutralino NLSP with Gravitino Dark Matter vs. Big Bang Nucleosynthesis, DESY-THESIS-2009-016 [SPIRES].

[19] A. Ferrantelli, Gravitino production from electroweak gauge boson scattering, JHEP 01 (2009) 070 [arXiv:0712.2171] [SPIRES].

[20] K. Kohri, Primordial nucleosynthesis and hadronic decay of a massive particle with a relatively short lifetime, Phys. Rev. D 64 (2001) 043515 [astro-ph/0103411] [SPIRES].

[21] M. Kawasaki, K. Kohri and T. Moroi, Big-bang nucleosynthesis and hadronic decay of long-lived massive particles, Phys. Rev. D 71 (2005) 083502 [astro-ph/0408426] [SPIRES]; K. Jedamzik, Did something decay, evaporate, or annihilate during big bang nucleosynthesis?, Phys. Rev. D 70 (2004) 063524 [astro-ph/0402344] [SPIRES].

[22] G. Jungman, M. Kamionkowski and K. Griest, Supersymmetric dark matter, Phys. Rept. 267 (1996) 195 [hep-ph/9506380] [SPIRES].

[23] G. Bélanger, F. Boudjema, A. Pukhov and A. Semenov, Dark matter direct detection rate in a generic model with MicrOMEGAs2.1, Comput. Phys. Commun. 180 (2009) 747 [arXiv:0803.2360] [SPIRES];

G. Bélanger, F. Boudjema, A. Pukhov and A. Semenov, MicrOMEGAs: Version 1.3, Comput. Phys. Commun. 174 (2006) 577 [hep-ph/0405253] [SPIRES]; MicrOMEGAs: A program for calculating the relic density in the MSSM, Comput. Phys. Commun. 149 (2002) 103 [hep-ph/0112278] [SPIRES].

[24] B.C. Allanach, SOFTSUSY: A C++ program for calculating supersymmetric spectra, Comput. Phys. Commun. 143 (2002) 305 [hep-ph/0104145] [SPIRES].

[25] LEPSUSYWG, ALEPH, DELPHI, L3 and OPAL experiments, note LEPSUSYWG/01-03.1 and note LEPSUSYWG/02-04.1, http://lepsusy.web.cern.ch/lepsusy/Welcome.html.

[26] M. Fujii, M. Ibe and T. Yanagida, Upper bound on gluino mass from thermal leptogenesis, Phys. Lett. B 579 (2004) 6 [hep-ph/0310142] [SPIRES].

[27] S. Blanchet and P. Di Bari, Flavor effects on leptogenesis predictions, JCAP 03 (2007) 018 [hep-ph/0607330] [SPIRES].

[28] M. Ibe and R. Kitano, Sweet Spot Supersymmetry, JHEP 08 (2007) 016 [arXiv:0705.3686] [SPIRES];

J.L. Feng, B.T. Smith and F. Takayama, Goldilocks Supersymmetry: Simultaneous Solution to Dark Matter and Flavor Problems of Supersymmetry, Phys. Rev. Lett. 100 (2008) 021302 [arXiv: 0709.0297] [SPIRES].

[29] R.H. Cyburt et al., Nucleosynthesis Constraints on a Massive Gravitino in Neutralino Dark Matter Scenarios, arXiv:0907.5003 [SPIRES]. 